\title{
Dual Polar Graphs, a nil-DAHA of Rank One, and Non-Symmetric Dual $q$-Krawtchouk Polynomials
}

\author{
Jae-Ho LEE ${ }^{\dagger}$ and Hajime TANAKA $\ddagger$ \\ $\dagger$ Department of Mathematics and Statistics, University of North Florida, \\ Jacksonville, FL 32224, USA \\ E-mail: jaeho.lee@unf.edu \\ URL: https://sites.google.com/site/jaeholeemath/ \\ $\ddagger$ Research Center for Pure and Applied Mathematics, Graduate School of Information Sciences, \\ Tohoku University, Sendai 980-8579, Japan \\ E-mail: htanaka@tohoku.ac.jp \\ URL: http://www.math.is.tohoku.ac.jp/ htanaka/
}

Received September 25, 2017, in final form January 29, 2018; Published online February 10, 2018

https://doi.org/10.3842/SIGMA.2018.009

\begin{abstract}
Let $\Gamma$ be a dual polar graph with diameter $D \geqslant 3$, having as vertices the maximal isotropic subspaces of a finite-dimensional vector space over the finite field $\mathbb{F}_{q}$ equipped with a non-degenerate form (alternating, quadratic, or Hermitian) with Witt index $D$. From a pair of a vertex $x$ of $\Gamma$ and a maximal clique $C$ containing $x$, we construct a $2 D$-dimensional irreducible module for a nil-DAHA of type $\left(C_{1}^{\vee}, C_{1}\right)$, and establish its connection to the generalized Terwilliger algebra with respect to $x, C$. Using this module, we then define the non-symmetric dual $q$-Krawtchouk polynomials and derive their recurrence and orthogonality relations from the combinatorial points of view. We note that our results do not depend essentially on the particular choice of the pair $x, C$, and that all the formulas are described in terms of $q, D$, and one other scalar which we assign to $\Gamma$ based on the type of the form.
\end{abstract}

Key words: dual polar graph; nil-DAHA; dual $q$-Krawtchouk polynomial; Terwilliger algebra; Leonard system

2010 Mathematics Subject Classification: 05E30; 20C08; 33D45; 33D80

\section{Introduction}

Q-polynomial distance-regular graphs are viewed as finite analogues of compact symmetric spaces of rank one, and have been extensively studied; cf. [1, 2, 10, 11]. By a famous theorem of Leonard [19], [1, Section 3.5], the duality property of $Q$-polynomial distance-regular graphs characterizes the terminating branch of the Askey scheme [14] of (basic) hypergeometric orthogonal polynomials, at the top (i.e., ${ }_{4} \phi_{3}$ ) of which are the $q$-Racah polynomials. A central tool in studying such a graph is the Terwilliger algebra $T=T(x)[31,32,33]$, which is a noncommutative semisimple matrix $\mathbb{C}$-algebra attached to every vertex $x$ of the graph.

Cherednik [3, 4, 5, 6] introduced the double affine Hecke algebras (DAHAs) for reduced affine root systems and used them to prove several conjectures for the Macdonald polynomials. Sahi [25] then extended the concept to the non-reduced affine root systems of type $\left(C_{n}^{\vee}, C_{n}\right)$ and proved the duality conjecture and other conjectures for the Koornwinder polynomials, which are the Macdonald polynomials attached to the affine root systems of type $\left(C_{n}^{\vee}, C_{n}\right)$. For $n=1$, these polynomials are the Askey-Wilson polynomials which are of ${ }_{4} \phi_{3}$, and the $q$-Racah polynomials are a discretization of the Askey-Wilson polynomials. 
Recently, the first author [16] found a link between the theories of $Q$-polynomial distanceregular graphs and the DAHAs. Namely, he considered a $Q$-polynomial distance-regular graph $\Gamma$ corresponding to $q$-Racah polynomials. He further assumed that $\Gamma$ possesses a clique $C$ with maximal possible size (called a Delsarte clique), and defined a semisimple matrix $\mathbb{C}$-algebra $\mathbf{T}=\mathbf{T}(x, C)$ attached to $C$ and a vertex $x \in C$, which contains $T(x)$ as a subalgebra. Then he showed that the so-called primary $\mathbf{T}$-module has the structure of an irreducible module for the DAHA of type $\left(C_{1}^{\vee}, C_{1}\right)$, and studied how the two module structures are related. In the subsequent paper [17], he captured in this context what should be called the non-symmetric q-Racah polynomials, which are the finite counterpart of the non-symmetric Askey-Wilson polynomials discussed by Sahi [25], and succeeded in giving an explicit combinatorial description of their orthogonality relations. We note that the expression for the non-symmetric $q$-Racah polynomials in [17] agrees with the one for the non-symmetric Askey-Wilson polynomials given by Koornwinder and Bouzeffour [15].

A big goal in this project is to establish a "non-symmetric version" of Leonard's theorem mentioned above. As the next attempt towards this goal, we discuss the dual polar graphs in this paper, and specialize the above situation to this case. The dual polar graphs are a classical family of $Q$-polynomial distance-regular graphs arising naturally as homogeneous spaces of finite classical groups by maximal parabolic subgroups, and correspond to the dual $q$-Krawtchouk polynomials which are of ${ }_{3} \phi_{2} ;$ cf. $[26,27]$. In particular, we will obtain the non-symmetric dual $q$-Krawtchouk polynomials and describe their recurrence and orthogonality relations; cf. Theorems 9.5 and 10.7. There are multiple motivations for the present work. First, for the $q$-Racah case, there is indeed no known example of a $Q$-polynomial distance-regular graph with large diameter (say, at least ten) having such a maximal clique, except the ordinary polygons with even order, which we view as rather trivial. Hence we may say that the theory developed in $[16,17]$ still remains somewhat at the algebraic/parametric level, whereas we will deal with concrete (and non-trivial) combinatorial examples in this paper. Second, there are of course other candidates of examples to be considered, such as the Grassmann graphs corresponding to the dual $q$-Hahn polynomials which lie in between the $q$-Racah and the dual $q$-Krawtchouk polynomials, but we decided to focus on the dual polar graphs, mainly because they exhibit quite a strong regularity of being regular near polygons, so that the computations become far simpler than those in $[16,17]$. Though many of our results can also be obtained in principle by taking appropriate limits of the (much involved) results in $[16,17]$, this fact motivates us to work out the details for this particular case rather independently of $[16,17]$. Third, we will encounter a nil$D A H A$ of type $\left(C_{1}^{\vee}, C_{1}\right)$, which is obtained by specializing some of the defining relations of the DAHA of type $\left(C_{1}^{\vee}, C_{1}\right)$. The nil-DAHAs were introduced and discussed recently by Cherednik and Orr [7, 8, 9], and our results demonstrate the fundamental importance of the concept in the theory of $Q$-polynomial distance-regular graphs; cf. Theorems 8.13 and 8.14. We may remark that our results are also relevant to recent work by Mazzocco [21, 22]; cf. Remarks 8.4 and 8.15.

The layout of the paper is as follows. In Section 2, we recall some background concerning $Q$-polynomial distance-regular graphs and their Terwilliger algebras. In Section 3, we recall the dual polar graphs and their properties. We fix a dual polar graph $\Gamma$ with diameter $D \geqslant 3$, having as vertices the maximal isotropic subspaces of a finite-dimensional vector space over the finite field $\mathbb{F}_{q}$ equipped with a non-degenerate form (alternating, quadratic, or Hermitian) with Witt index $D$. We fix a maximal clique $C$ of $\Gamma$, and discuss the Terwilliger algebra of $\Gamma$ with respect to $C$ in the sense of Suzuki [28]. In Section 4, we also fix a vertex $x \in C$ and define the generalized Terwilliger algebra $\mathbf{T}=\mathbf{T}(x, C)$ of $\Gamma$. We then define from $x$ and $C$ a $2 D$-dimensional subspace $\mathbf{W}$ of the standard module for $\mathbf{T}$, and show that it is an irreducible $\mathbf{T}$-module. In Section 5, we recall the basic theory concerning Leonard systems, a certain linear algebraic framework for Leonard's theorem introduced by Terwilliger [34]. We focus on the class of Leonard systems corresponding to the dual $q$-Krawtchouk polynomials and discuss their properties. To 
the $\mathbf{T}$-module $\mathbf{W}$ we attach four Leonard systems, all of which belong to this class, and we study each of these Leonard systems in detail in Sections 6 and 7. In Section 8, we introduce a nil-DAHA $\overline{\mathcal{H}}$ of type $\left(C_{1}^{\vee}, C_{1}\right)$, and define a $2 D$-dimensional representation $\overline{\mathcal{H}} \rightarrow \operatorname{End}(\mathbf{W})$. Our first main results of this paper describe how the $\mathbf{T}$-action on $\mathbf{W}$ is related to the $\overline{\mathcal{H}}$-action; cf. Theorems 8.13 and 8.14. In Section 9, we define the non-symmetric dual $q$-Krawtchouk polynomials $\ell_{i}^{ \pm}$using the representation $\overline{\mathcal{H}} \rightarrow \operatorname{End}(\mathbf{W})$ and discuss a role of these Laurent polynomials in $\mathbf{W}$. In fact, we will obtain two expressions for the $\ell_{i}^{ \pm}$. Recurrence relations involving at most four terms will also be given; cf. Theorem 9.5. The standard (Hermitian) inner product on $\mathbf{W}$ gives rise to an inner product on the $2 D$-dimensional vector space to which the $\ell_{i}^{ \pm}$belong, which ultimately leads in Section 10 to our second main result of this paper, i.e., a combinatorial description of the orthogonality relations for the $\ell_{i}^{ \pm}$; cf. Theorem 10.7 . We note that our results do not depend essentially on the particular choice of the pair $x, C$, and that all the formulas are described in terms of $q, D$, and one other scalar $e$ which we assign to $\Gamma$ based on the type of the non-degenerate form.

Throughout this paper, we use the following notation. For a given non-empty finite set $X$, let $\operatorname{Mat}_{X}(\mathbb{C})$ be the $\mathbb{C}$-algebra consisting of the complex square matrices indexed by $X$. Let $V=V_{X}$ be the $\mathbb{C}$-vector space consisting of the complex column vectors indexed by $X$. We endow $V$ with the inner product $\langle u, v\rangle=u^{t} \bar{v}$ for $u, v \in V$, where ${ }^{t}$ denotes transpose and ${ }^{-}$denotes complex conjugate. We abbreviate $\|u\|^{2}=\langle u, u\rangle$ for all $u \in V$. For every $y \in X$, let $\hat{y}$ be the vector in $V$ with a 1 in the $y$-coordinate and 0 elsewhere. For a subset $Y \subset X$, let $\hat{Y}=\sum_{y \in Y} \hat{y} \in V$ denote its (column) characteristic vector. A Laurent polynomial $f(\eta) \in \mathbb{C}\left[\eta, \eta^{-1}\right]$ in the variable $\eta$ is said to be symmetric if $f(\eta)=f\left(\eta^{-1}\right)$, and non-symmetric otherwise. Note that the symmetric Laurent polynomials are precisely the polynomials in $\xi:=\eta+\eta^{-1}$. Let $q$ be a prime power. For $r \in \mathbb{C}$ and an integer $n \geqslant 0$, let

$$
(r ; q)_{n}=(1-r)(1-r q) \cdots\left(1-r q^{n-1}\right), \quad\left[\begin{array}{c}
n \\
1
\end{array}\right]=\left[\begin{array}{c}
n \\
1
\end{array}\right]_{q}=\frac{q^{n}-1}{q-1} .
$$

For $r_{1}, r_{2}, \ldots, r_{k+1}, s_{1}, s_{2}, \ldots, s_{k} \in \mathbb{C}$, let

$$
{ }_{k+1} \phi_{k}\left(\begin{array}{c}
r_{1}, r_{2}, \ldots, r_{k+1} \\
s_{1}, s_{2}, \ldots, s_{k}
\end{array} \mid q, \eta\right)=\sum_{n=0}^{\infty} \frac{\left(r_{1} ; q\right)_{n}\left(r_{2} ; q\right)_{n} \cdots\left(r_{k+1} ; q\right)_{n}}{\left(s_{1} ; q\right)_{n}\left(s_{2} ; q\right)_{n} \cdots\left(s_{k} ; q\right)_{n}} \frac{\eta^{n}}{(q ; q)_{n}} .
$$

\section{Distance-regular graphs}

Let $\Gamma$ be a finite simple connected graph with vertex set $X$ and diameter $D$. For $x \in X$, let

$$
\Gamma_{i}(x)=\{y \in X: \partial(x, y)=i\}, \quad 0 \leqslant i \leqslant D,
$$

where $\partial$ denotes the path-length distance. We abbreviate $\Gamma(x):=\Gamma_{1}(x)$. We call $\Gamma$ distanceregular if there are non-negative integers $a_{i}, b_{i}, c_{i}, 0 \leqslant i \leqslant D$, called the intersection numbers of $\Gamma$, such that $b_{D}=c_{0}=0, b_{i-1} c_{i} \neq 0,1 \leqslant i \leqslant D$, and

$$
a_{i}=\left|\Gamma_{i}(x) \cap \Gamma(y)\right|, \quad b_{i}=\left|\Gamma_{i+1}(x) \cap \Gamma(y)\right|, \quad c_{i}=\left|\Gamma_{i-1}(x) \cap \Gamma(y)\right|
$$

for every pair of vertices $x, y \in X$ with $\partial(x, y)=i$, where $\Gamma_{-1}(x)=\Gamma_{D+1}(x):=\varnothing$. Fig. 1 shows a small example of a distance-regular graph with $D=3$.

From now on, assume that $\Gamma$ is distance-regular. The $i^{\text {th }}$ distance matrix of $\Gamma$ is the $0-1$ matrix $A_{i} \in \operatorname{Mat}_{X}(\mathbb{C})$ such that $\left(A_{i}\right)_{x, y}=1$ if and only if $\partial(x, y)=i$. The Bose-Mesner algebra of $\Gamma$ is the subalgebra $M$ of $\operatorname{Mat}_{X}(\mathbb{C})$ generated by the $A_{i}$. We note that $M$ is semisimple since it is closed under conjugate-transposition. Observe also that

$$
A_{1} A_{i}=b_{i-1} A_{i-1}+a_{i} A_{i}+c_{i+1} A_{i+1}, \quad 0 \leqslant i \leqslant D,
$$




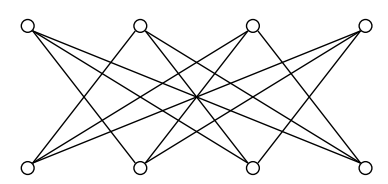

Figure 1. The complement of the $2 \times 4$-grid.

from which it follows that, for $0 \leqslant i \leqslant D$, there is a polynomial $v_{i} \in \mathbb{C}[\xi]$ with $\operatorname{deg}\left(v_{i}\right)=i \operatorname{such}$ that $v_{i}\left(A_{1}\right)=A_{i}$. It follows that the adjacency matrix $A:=A_{1}$ of $\Gamma$ generates $M$, and that the $A_{i}$ form a basis for $M$. In particular, we have $\operatorname{dim}(M)=D+1$.

Since $A$ is real symmetric and generates $M$, it has $D+1$ mutually distinct real eigenvalues $\theta_{0}, \theta_{1}, \ldots, \theta_{D}$, which we call the eigenvalues of $\Gamma$. We will always set $\theta_{0}:=b_{0}$, the valency (or degree) of $\Gamma$. For $0 \leqslant i \leqslant D$, let $E_{i} \in \operatorname{Mat}_{X}(\mathbb{C})$ be the orthogonal projection onto the eigenspace of $\theta_{i}$. Then we have

$$
A=\sum_{i=0}^{D} \theta_{i} E_{i},
$$

and the $E_{i}$ form another basis for $M$ consisting of the primitive idempotents, i.e., $E_{i} E_{j}=\delta_{i, j} E_{i}$, $\sum_{i=0}^{D} E_{i}=I$ (the identity matrix). We note that $E_{0} V=\mathbb{C} \hat{X}$. Observe that $M$ is also closed under entrywise multiplication, denoted $\circ$. We say that $\Gamma$ is $Q$-polynomial with respect to the ordering $\left\{E_{i}\right\}_{i=0}^{D}\left(\right.$ or $\left.\left\{\theta_{i}\right\}_{i=0}^{D}\right)$ if there are scalars $a_{i}^{*}, b_{i}^{*}, c_{i}^{*}, 0 \leqslant i \leqslant D$, such that $b_{D}^{*}=c_{0}^{*}=0, b_{i-1}^{*} c_{i}^{*} \neq 0$, $1 \leqslant i \leqslant D$, and

$$
E_{1} \circ E_{i}=\frac{1}{|X|}\left(b_{i-1}^{*} E_{i-1}+a_{i}^{*} E_{i}+c_{i+1}^{*} E_{i+1}\right), \quad 0 \leqslant i \leqslant D,
$$

where we set $b_{-1}^{*} E_{-1}=c_{D+1}^{*} E_{D+1}:=0$. If this is the case, then for $0 \leqslant i \leqslant D$, there is a polynomial $v_{i}^{*} \in \mathbb{C}[\xi]$ with $\operatorname{deg}\left(v_{i}^{*}\right)=i$ such that $v_{i}^{*}\left(|X| E_{1}\right)=|X| E_{i}$, where the multiplication is under $\circ$. In particular, if we write

$$
E_{1}=\frac{1}{|X|} \sum_{i=0}^{D} \theta_{i}^{*} A_{i},
$$

then the $\theta_{i}^{*}$ are (real and) mutually distinct. Note also that $\theta_{0}^{*}=\operatorname{trace}\left(E_{1}\right)=\operatorname{rank}\left(E_{1}\right)$.

Assume that $\Gamma$ is $Q$-polynomial with respect to the ordering $\left\{E_{i}\right\}_{i=0}^{D}$. Fix a 'base vertex' $x \in X$. For $0 \leqslant i \leqslant D$, let $E_{i}^{*}=E_{i}^{*}(x):=\operatorname{diag}\left(A_{i} \hat{x}\right) \in \operatorname{Mat}_{X}(\mathbb{C})$. Note that $E_{i}^{*} E_{j}^{*}=\delta_{i, j} E_{i}^{*}$, $\sum_{i=0}^{D} E_{i}^{*}=I$. The $E_{i}^{*}$ form a basis for the dual Bose-Mesner algebra $M^{*}=M^{*}(x)$ of $\Gamma$ with respect to $x$. We call $A^{*}=A^{*}(x):=|X| \operatorname{diag}\left(E_{1} \hat{x}\right) \in \operatorname{Mat}_{X}(\mathbb{C})$ the dual adjacency matrix of $\Gamma$ with respect to $x$. We have

$$
A^{*}=\sum_{i=0}^{D} \theta_{i}^{*} E_{i}^{*},
$$

so that $A^{*}$ generates $M^{*}$, and that the $\theta_{i}^{*}$ are the eigenvalues of $A^{*}$, which we call the dual eigenvalues of $\Gamma$. The Terwilliger (or subconstituent) algebra $T=T(x)$ with respect to $x$ is the subalgebra of $\operatorname{Mat}_{X}(\mathbb{C})$ generated by $M, M^{*}[31,32,33]$. We note that $T$ is again semisimple, is generated by $A, A^{*}$, and that any two non-isomorphic irreducible $T$-modules in $V$ are orthogonal. The following are relations in $T$ (cf. [31, Lemma 3.2]):

$$
E_{i}^{*} A E_{j}^{*}=E_{i} A^{*} E_{j}=0 \quad \text { if }|i-j|>1, \quad 0 \leqslant i, j \leqslant D .
$$


Observe also that $E_{i}^{*} \hat{X}=A_{i} \hat{x}, 0 \leqslant i \leqslant D$, from which it follows that the $(D+1)$-dimensional subspace

$$
M \hat{x}=M^{*} \hat{X}=\bigoplus_{i=0}^{D} \mathbb{C} A_{i} \hat{x}=\bigoplus_{i=0}^{D} \mathbb{C} E_{i} \hat{x}
$$

of $V$ is an irreducible $T$-module, called the primary $T$-module. We note that

$$
A . A_{i} \hat{x}=b_{i-1} A_{i-1} \hat{x}+a_{i} A_{i} \hat{x}+c_{i+1} A_{i+1} \hat{x}, \quad A^{*} . A_{i} \hat{x}=\theta_{i}^{*} A_{i} \hat{x}, \quad 0 \leqslant i \leqslant D .
$$

We refer the reader to $[1,2,10,11]$ for more detailed information.

\section{Dual polar graphs}

Let $D$ be a positive integer. Let $\mathbb{V}$ be one of the following spaces over the finite field $\mathbb{F}_{q}$ equipped with a non-degenerate form: ${ }^{1}$

\begin{tabular}{cccc}
\hline space & dimension & form & $e$ \\
\hline \hline$\left[C_{D}(q)\right]$ & $2 D$ & alternating & 1 \\
{$\left[B_{D}(q)\right]$} & $2 D+1$ & quadratic & 1 \\
{$\left[D_{D}(q)\right]$} & $2 D$ & quadratic (maximal Witt index $D)$ & 0 \\
{$\left[{ }^{2} D_{D+1}(q)\right]$} & $2 D+2$ & quadratic (non-maximal Witt index $D)$ & 2 \\
{$\left[{ }^{2} A_{2 D}(r)\right]$} & $2 D+1$ & Hermitian $\left(q=r^{2}\right)$ & $\frac{3}{2}$ \\
{$\left[{ }^{2} A_{2 D-1}(r)\right]$} & $2 D$ & Hermitian $\left(q=r^{2}\right)$ & $\frac{1}{2}$ \\
\hline
\end{tabular}

A subspace of $\mathbb{V}$ is called (totally) isotropic if the form vanishes completely on it. We note that maximal isotropic subspaces have dimension $D$. Let $X$ be the set of all maximal isotropic subspaces of $\mathbb{V}$. The dual polar graph (on $\mathbb{V}$ ) has vertex set $X$, where two vertices $x, y$ are adjacent if and only if $\operatorname{dim}(x \cap y)=D-1$; cf. [2, Section 9.4]. We have $\partial(x, y)=D-\operatorname{dim}(x \cap y)$ in general, so that the diameter equals $D$.

Assumption 3.1. For the rest of this paper, we will always assume that $\Gamma$ is a dual polar graph with diameter $D \geqslant 3$.

First we summarize some results that we need. The graph $\Gamma$ is distance-regular with intersection numbers

$$
a_{i}=\left(q^{e}-1\right)\left[\begin{array}{l}
i \\
1
\end{array}\right], \quad b_{i}=q^{i+e}\left[\begin{array}{c}
D-i \\
1
\end{array}\right], \quad c_{i}=\left[\begin{array}{l}
i \\
1
\end{array}\right], \quad 0 \leqslant i \leqslant D .
$$

Note that $a_{i}=c_{i} a_{1}, 0 \leqslant i \leqslant D$. The eigenvalues of $\Gamma$ are given by

$$
\theta_{i}=q^{e}\left[\begin{array}{c}
D-i \\
1
\end{array}\right]-\left[\begin{array}{l}
i \\
1
\end{array}\right], \quad 0 \leqslant i \leqslant D
$$

and $\Gamma$ is $Q$-polynomial with respect to the ordering ${ }^{2}\left\{\theta_{i}\right\}_{i=0}^{D}$, where $\theta_{0}>\theta_{1}>\cdots>\theta_{D}$. Moreover, the corresponding dual eigenvalues are given by

$$
\theta_{i}^{*}=\frac{q\left(1+q^{D+e-2}\right)}{1-q}+\frac{q\left(1+q^{D+e-2}\right)\left(1+q^{D+e-1}\right)}{(q-1)\left(1+q^{e-1}\right)} q^{-i}, \quad 0 \leqslant i \leqslant D .
$$

\footnotetext{
${ }^{1}$ The scalar $e$ is from [2, Section 9.4] and is assigned to $\mathbb{V}$ to give unified descriptions to various formulas regarding the dual polar spaces.

${ }^{2}$ The dual polar graph on $\left[{ }^{2} A_{2 D-1}(r)\right]$ has another $Q$-polynomial ordering, which is $\theta_{0}, \theta_{D}, \theta_{1}, \theta_{D-1}, \ldots$ in terms of the natural ordering $\theta_{0}>\theta_{1}>\cdots>\theta_{D}$; cf. [1, p. 304]. However, it turns out that the maximal cliques do not behave well with this ordering (cf. [30, Theorem 8.8]), so that we will not pay attention to it in this paper.
} 
See [2, Theorem 9.4.3] and [37, Lemma 16.5]. The dual polar graph $\Gamma$ is an example of a regular near polygon (cf. $[2$, Section 6.4$]$ ), which means that $\Gamma$ does not have

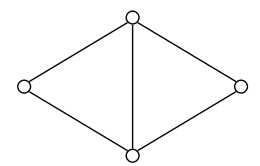

(i.e., $\left.K_{1,1,2}\right)$ as an induced subgraph, and that for every $y \in X$ and a maximal clique $C$, there is a unique $z \in C$ nearest to $y$, provided that $\partial(y, C)<D$. Note that the former condition implies that every edge lies in a unique maximal clique. We also note that $\Gamma$ is more specifically a regular near $2 D$-gon, ${ }^{3}$ i.e., there is in fact no vertex $y$ at distance $D$ from $C$.

Let $C$ be a maximal clique in $\Gamma$. By the above comments, we have

$$
|C|=a_{1}+2=1+q^{e},
$$

which attains the Hoffman bound $1-\theta_{0} \theta_{D}^{-1}$ (cf. [2, Proposition 4.4.6]). In other words, $C$ is a so-called Delsarte clique. For $0 \leqslant i \leqslant D-1$, define the $i^{\text {th }}$ distance neighbor of $C$ by

$$
C_{i}=\{y \in X: \partial(y, C)=i\}
$$

Then we have

$$
A_{i} \hat{C}=\hat{C}_{i}+q^{e} \hat{C}_{i-1}, \quad 0 \leqslant i \leqslant D,
$$

where $C_{-1}=C_{D}:=\varnothing$, from which it follows that

$$
M \hat{C}=\bigoplus_{i=0}^{D-1} \mathbb{C} \hat{C}_{i}
$$

In other words, $\left\{C_{i}\right\}_{i=0}^{D-1}$ is an equitable partition of $X$. In particular, there are non-negative integers $\widetilde{a}_{i}, \widetilde{b}_{i}, \widetilde{c}_{i}, 0 \leqslant i \leqslant D-1$, called the intersection numbers of $C$, such that $\widetilde{b}_{D-1}=\widetilde{c}_{0}=0$, $\widetilde{b}_{i-1} \widetilde{c}_{i} \neq 0,1 \leqslant i \leqslant D-1$, and

$$
\widetilde{a}_{i}=\left|C_{i} \cap \Gamma(y)\right|, \quad \widetilde{b}_{i}=\left|C_{i+1} \cap \Gamma(y)\right|, \quad \widetilde{c}_{i}=\left|C_{i-1} \cap \Gamma(y)\right|
$$

for every $y \in C_{i}$. We may remark that a clique in a distance-regular graph satisfies (3.6) precisely when it is a Delsarte clique; cf. [11, Section 13.7].

Lemma 3.2. The following (i), (ii) hold:

(i) $\widetilde{c}_{i}=c_{i}, 1 \leqslant i \leqslant D-1$.

(ii) $\widetilde{b}_{i}=b_{i+1}, 0 \leqslant i \leqslant D-2$.

Proof. (i) Let $y \in C_{i}$. Recall that there is a unique $z$ in $C \cap \Gamma_{i}(y)$ since $\Gamma$ is a regular near polygon. Then we have $C_{i-1} \cap \Gamma(y)=\Gamma_{i-1}(z) \cap \Gamma(y)$, and the result follows.

(ii) With the above notation, pick any $z^{\prime} \in C$ with $z^{\prime} \neq z$. Then $z^{\prime} \in \Gamma_{i+1}(y)$, and we have $C_{i+1} \cap \Gamma(y)=\Gamma_{i+2}\left(z^{\prime}\right) \cap \Gamma(y)$, as desired.

Using (3.1), Lemma 3.2 , and $\widetilde{a}_{i}+\widetilde{b}_{i}+\widetilde{c}_{i}=\theta_{0}$, we have

$$
\widetilde{a}_{i}=q^{e}\left[\begin{array}{c}
i+1 \\
1
\end{array}\right]-\left[\begin{array}{l}
i \\
1
\end{array}\right], \quad \widetilde{b}_{i}=q^{i+1+e}\left[\begin{array}{c}
D-i-1 \\
1
\end{array}\right], \quad \widetilde{c}_{i}=\left[\begin{array}{l}
i \\
1
\end{array}\right], \quad 0 \leqslant i \leqslant D-1 .
$$

\footnotetext{
${ }^{3}$ The identity $a_{i}=c_{i} a_{1}, 0 \leqslant i \leqslant D$, mentioned above is a consequence of this; cf. [2, Theorem 6.4.1].
} 
We now recall the Terwilliger algebra of $\Gamma$ with respect to $C$ in the sense of Suzuki [28]. For $0 \leqslant i \leqslant D-1$, let $\widetilde{E}_{i}^{*}=\widetilde{E}_{i}^{*}(C):=\operatorname{diag}\left(\hat{C}_{i}\right) \in \operatorname{Mat}_{X}(\mathbb{C})$. Note that $\widetilde{E}_{i}^{*} \widetilde{E}_{j}^{*}=\delta_{i, j} \widetilde{E}_{i}^{*}, \sum_{i=0}^{D-1} \widetilde{E}_{i}^{*}=I$. The $\widetilde{E}_{i}^{*}$ form a basis for the dual Bose-Mesner algebra $\widetilde{M}^{*}=\widetilde{M}^{*}(C)$ of $\Gamma$ with respect to $C$. The dual adjacency matrix of $\Gamma$ with respect to $C$ is defined by (cf. [16, equation (50)])

$$
\widetilde{A}^{*}=\widetilde{A}^{*}(C):=\frac{|X|}{|C|} \operatorname{diag}\left(E_{1} \hat{C}\right)=\frac{1}{|C|} \sum_{y \in C} A^{*}(y) .
$$

From (2.1), (3.4), and (3.5), it follows that

$$
\frac{1}{|C|} E_{1} \hat{C}=\frac{1}{|X|} \sum_{i=0}^{D-1} \widetilde{\theta}_{i}^{*} \hat{C}_{i}
$$

where

$$
\widetilde{\theta}_{i}^{*}=\frac{1}{1+q^{e}} \theta_{i}^{*}+\frac{q^{e}}{1+q^{e}} \theta_{i+1}^{*}, \quad 0 \leqslant i \leqslant D-1,
$$

so that (cf. [16, Lemma 4.11])

$$
\widetilde{A}^{*}=\sum_{i=0}^{D-1} \widetilde{\theta}_{i}^{*} \widetilde{E}_{i}^{*}
$$

By (3.3), we have

$$
\widetilde{\theta}_{i}^{*}=\frac{q\left(1+q^{D+e-2}\right)}{1-q}+\frac{q\left(1+q^{D+e-2}\right)\left(1+q^{D+e-1}\right)}{(q-1)\left(1+q^{e}\right)} q^{-i}, \quad 0 \leqslant i \leqslant D-1 .
$$

In particular, $\widetilde{A}^{*}$ has $D$ mutually distinct real eigenvalues and hence generates $\widetilde{M}^{*}$. The Terwilliger algebra $\widetilde{T}=\widetilde{T}(C)$ with respect to $C$ is the subalgebra of $\operatorname{Mat}_{X}(\mathbb{C})$ generated by $M, \widetilde{M}^{*}$. We note that $\widetilde{T}$ is semisimple and is generated by $A, \widetilde{A}^{*}$. By virtue of $(2.2)$, the following are relations in $\widetilde{T}$ :

$$
\widetilde{E}_{i}^{*} A \widetilde{E}_{j}^{*}=E_{i} \widetilde{A}^{*} E_{j}=0 \quad \text { if }|i-j|>1, \quad 0 \leqslant i, j \leqslant D,
$$

where we set $\widetilde{E}_{D}^{*}:=0$ for convenience. The subspace (3.6) of $V$ is an irreducible $\widetilde{T}$-module with dimension $D$, called the primary $\widetilde{T}$-module. We note that

$$
A \cdot \hat{C}_{i}=\widetilde{b}_{i-1} \hat{C}_{i-1}+\widetilde{a}_{i} \hat{C}_{i}+\widetilde{c}_{i+1} \hat{C}_{i+1}, \quad \widetilde{A}^{*} \cdot \hat{C}_{i}=\widetilde{\theta}_{i}^{*} \hat{C}_{i}, \quad 0 \leqslant i \leqslant D-1 .
$$

Remark 3.3. The Bose-Mesner algebra $M$ coincides in this case with the commutant of the corresponding classical group acting on $X$, whereas the Terwilliger algebras $T, \widetilde{T}$ are subalgebras of those of maximal parabolic subgroups. See also [26, 27].

\section{The algebra $\mathrm{T}$}

We continue to discuss the dual polar graph $\Gamma$.

Assumption 4.1. For the rest of this paper, we will fix a vertex $x \in X$ and a maximal clique $C$ containing $x$. 


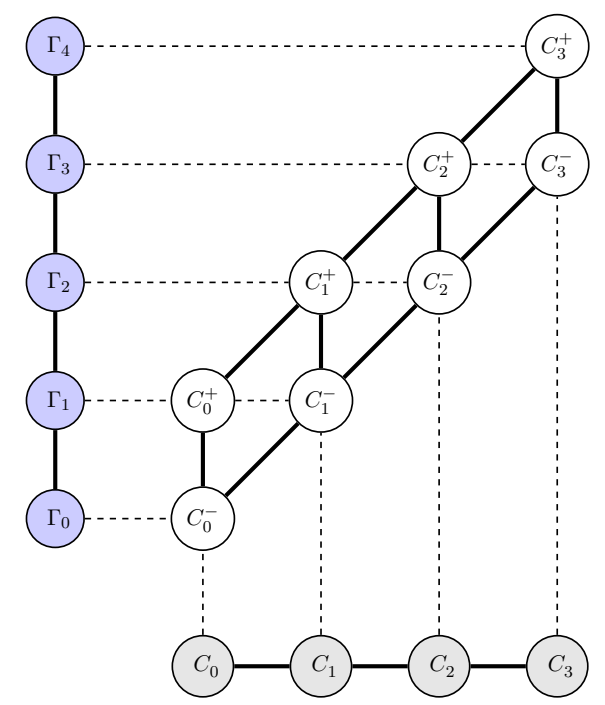

Figure 2. The partition $\left\{C_{i}^{ \pm}\right\}_{i=0}^{D-1}$ when $D=4$.

Definition 4.2 ([16, Definition 5.20]). The generalized Terwilliger algebra of $\Gamma$ with respect to $x, C$ is the subalgebra $\mathbf{T}=\mathbf{T}(x, C)$ of $\operatorname{Mat}_{X}(\mathbb{C})$ generated by $T=T(x), \widetilde{T}=\widetilde{T}(C)$.

We note that $\mathbf{T}$ is semisimple and is generated by $A, A^{*}$, and $\widetilde{A}^{*}$, where $A^{*} \widetilde{A}^{*}=\widetilde{A}^{*} A^{*}$. We now refine the equitable partition $\left\{C_{i}\right\}_{i=0}^{D-1}$ of $X$ as follows

$$
C_{i}^{-}=\Gamma_{i}(x) \cap C_{i}, \quad C_{i}^{+}=\Gamma_{i+1}(x) \cap C_{i}, \quad 0 \leqslant i \leqslant D-1 .
$$

See Fig. 2. For notational convenience, we set

$$
C_{-1}^{-}=C_{-1}^{+}=C_{D}^{-}=C_{D}^{+}:=\varnothing .
$$

Observe that $C_{i}=C_{i}^{-} \cup C_{i}^{+}, 0 \leqslant i \leqslant D-1$, and that $\Gamma_{i}(x)=C_{i-1}^{+} \cup C_{i}^{-}, 0 \leqslant i \leqslant D$.

Lemma 4.3. The following $(i)$, (ii) hold:

(i) For $0 \leqslant i \leqslant D-1$ and $z \in C_{i}^{-}$, we have

\begin{tabular}{c||ccccc}
$Y$ & $C_{i-1}^{-}$ & $C_{i-1}^{+}$ & $C_{i}^{-}$ & $C_{i}^{+}$ & $C_{i+1}^{-}$ \\
\hline$|\Gamma(z) \cap Y|$ & $c_{i}$ & 0 & $a_{i}$ & $b_{i}-b_{i+1}$ & $b_{i+1}$
\end{tabular}.

(ii) For $0 \leqslant i \leqslant D-1$ and $z \in C_{i}^{+}$, we have

\begin{tabular}{c||ccccc}
$Y$ & $C_{i-1}^{+}$ & $C_{i}^{-}$ & $C_{i}^{+}$ & $C_{i+1}^{-}$ & $C_{i+1}^{+}$ \\
\hline$|\Gamma(z) \cap Y|$ & $c_{i}$ & $c_{i+1}-c_{i}$ & $a_{i+1}$ & 0 & $b_{i+1}$
\end{tabular}.

In particular, the partition $\left\{C_{i}^{ \pm}\right\}_{i=0}^{D-1}$ is again equitable. ${ }^{4}$

Proof. Use Lemma 3.2.

Lemma 4.4. We have

$$
\left|C_{i}^{-}\right|=q^{i e} \prod_{n=1}^{i} \frac{q^{D}-q^{n}}{q^{n}-1}, \quad\left|C_{i}^{+}\right|=q^{(i+1) e} \prod_{n=1}^{i} \frac{q^{D}-q^{n}}{q^{n}-1}, \quad 0 \leqslant i \leqslant D-1 .
$$

In particular, the $C_{i}^{ \pm}$are non-empty.

\footnotetext{
${ }^{4}$ See [12, Proposition 1.3] for a more general result.
} 
Proof. It follows from Lemma 4.3 that

$$
c_{i}\left|C_{i}^{-}\right|=b_{i}\left|C_{i-1}^{-}\right|, \quad c_{i}\left|C_{i}^{+}\right|=b_{i}\left|C_{i-1}^{+}\right|
$$

The result follows from this and (3.1), together with $\left|C_{0}^{-}\right|=1$ and $\left|C_{0}^{+}\right|=q^{e}$ (cf. (3.4)).

Let $\mathbf{W}$ be the linear span of the $\hat{C}_{i}^{ \pm}$. Thus, $\mathbf{W}$ has the following ordered orthogonal basis:

$$
\mathcal{C}=\left\{\hat{C}_{0}^{-}, \hat{C}_{0}^{+}, \hat{C}_{1}^{-}, \hat{C}_{1}^{+}, \ldots, \hat{C}_{D-1}^{-}, \hat{C}_{D-1}^{+}\right\}
$$

Proposition 4.5. The subspace $\mathbf{W}$ is an irreducible $\mathbf{T}$-module.

Proof. It is clear that $\mathbf{W}$ is closed under the actions of the $E_{i}^{*}$ and the $\widetilde{E}_{i}^{*}$. Moreover, since $\left\{C_{i}^{ \pm}\right\}_{i=0}^{D-1}$ is an equitable partition by Lemma $4.3, \mathbf{W}$ is also $A$-invariant. It follows that $\mathbf{W}$ is a $\mathbf{T}$-module. We now show the irreducibility. Since $\mathbf{T}$ is semisimple, $\mathbf{W}$ is an orthogonal direct sum of irreducible $\mathbf{T}$-modules. Among these $\mathbf{T}$-modules, there is one which is not orthogonal to $\mathbb{C} \hat{X}=E_{0} \mathbf{W}$, denoted by $\mathbf{W}_{0}$. Then we have $0 \neq E_{0} \mathbf{W}_{0} \subset E_{0} \mathbf{W}$, so that $\hat{X} \in E_{0} \mathbf{W}_{0} \subset \mathbf{W}_{0}$, from which it follows that $\mathbf{W}=M^{*} \widetilde{M}^{*} \hat{X} \subset \mathbf{W}_{0}$. Hence $\mathbf{W}_{0}=\mathbf{W}$, and the result follows.

We call $\mathbf{W}$ the primary $\mathbf{T}$-module. Note that $\mathbf{W}$ contains both the primary $T$-module $M \hat{x}$ and the primary $\widetilde{T}$-module $M \hat{C}$. Let $M \hat{x}^{\perp}$ (resp. $M \hat{C}^{\perp}$ ) be the orthogonal complement of $M \hat{x}$ (resp. $M \hat{C})$ in $\mathbf{W}$. In Sections 6 and 7, we will show that $M \hat{x}^{\perp}$ (resp. $M \hat{C}^{\perp}$ ) is also an irreducible $T$-module (resp. $\widetilde{T}$-module). Thus, $\mathbf{W}$ decomposes in two ways:

$$
\begin{array}{rlr}
\mathbf{W} & =M \hat{x} \oplus M \hat{x}^{\perp} & \text { (orthogonal direct sum of irreducible } T \text {-modules) } \\
& =M \hat{C} \oplus M \hat{C}^{\perp} & \text { (orthogonal direct sum of irreducible } \widetilde{T} \text {-modules). }
\end{array}
$$

We end this section by describing the actions of $A, A^{*}$, and $\widetilde{A}^{*}$ on $\mathbf{W}$ in terms of the basis $\mathcal{C}$.

Lemma 4.6. For $0 \leqslant i \leqslant D-1$, we have

$$
\begin{aligned}
& A . \hat{C}_{i}^{-}=\frac{q^{D+e}-q^{i+e}}{q-1} \hat{C}_{i-1}^{-}+\left(q^{e}-1\right) \frac{q^{i}-1}{q-1} \hat{C}_{i}^{-}+q^{i} \hat{C}_{i}^{+}+\frac{q^{i+1}-1}{q-1} \hat{C}_{i+1}^{-}, \\
& A . \hat{C}_{i}^{+}=\frac{q^{D+e}-q^{i+e}}{q-1} \hat{C}_{i-1}^{+}+q^{e+i} \hat{C}_{i}^{-}+\left(q^{e}-1\right) \frac{q^{i+1}-1}{q-1} \hat{C}_{i}^{+}+\frac{q^{i+1}-1}{q-1} \hat{C}_{i+1}^{+} .
\end{aligned}
$$

Proof. From Lemma 4.3 it follows that

$$
\begin{aligned}
& A . \hat{C}_{i}^{-}=b_{i} \hat{C}_{i-1}^{-}+a_{i} \hat{C}_{i}^{-}+\left(c_{i+1}-c_{i}\right) \hat{C}_{i}^{+}+c_{i+1} \hat{C}_{i+1}^{-} \\
& A . \hat{C}_{i}^{+}=b_{i} \hat{C}_{i-1}^{+}+\left(b_{i}-b_{i+1}\right) \hat{C}_{i}^{-}+a_{i+1} \hat{C}_{i}^{+}+c_{i+1} \hat{C}_{i+1}^{+}
\end{aligned}
$$

for $0 \leqslant i \leqslant D-1$. Evaluate the two identities using (3.1).

Lemma 4.7. For $0 \leqslant i \leqslant D-1$, we have

$$
A^{*} . \hat{C}_{i}^{-}=\theta_{i}^{*} \hat{C}_{i}^{-}, \quad A^{*} . \hat{C}_{i}^{+}=\theta_{i+1}^{*} \hat{C}_{i}^{+}, \quad \widetilde{A}^{*} . \hat{C}_{i}^{ \pm}=\widetilde{\theta}_{i}^{*} \hat{C}_{i}^{ \pm}
$$

Proof. Clear. 


\section{Leonard systems of dual $q$-Krawtchouk type}

Let $d$ be a positive integer, and let $\mathrm{W}$ be a vector space over $\mathbb{C}$ with $\operatorname{dim}(\mathrm{W})=d+1$. An element $\mathrm{A} \in \operatorname{End}(\mathrm{W})$ is called multiplicity-free if it has $d+1$ mutually distinct eigenvalues. Suppose that $\mathrm{A}$ is multiplicity-free, and let $\left\{\theta_{i}\right\}_{i=0}^{d}$ be an ordering of the eigenvalues of $\mathrm{A}$. Then there is a sequence of elements $\left\{\mathrm{E}_{i}\right\}_{i=0}^{d}$ in $\operatorname{End}(\mathrm{W})$ such that $\mathrm{AE}_{i}=\theta_{i} \mathrm{E}_{i}, \mathrm{E}_{i} \mathrm{E}_{j}=\delta_{i, j} \mathrm{E}_{i}, \sum_{i=0}^{d} \mathrm{E}_{i}=\mathrm{I}$, where $\mathbf{I}$ is the identity of $\operatorname{End}(\mathrm{W})$. We call $\mathrm{E}_{i}$ the primitive idempotent of $\mathrm{A}$ associated with $\theta_{i}$.

Definition 5.1. A Leonard system on $\mathrm{W}$ is a sequence

$$
\Phi=\left(\mathrm{A} ; \mathrm{A}^{*} ;\left\{\mathrm{E}_{i}\right\}_{i=0}^{d} ;\left\{\mathrm{E}_{i}^{*}\right\}_{i=0}^{d}\right)
$$

that satisfies the following axioms (LS1)-(LS4):

(LS1) Each of $A, A^{*}$ is a multiplicity-free element in $\operatorname{End}(W)$.

(LS2) $\left\{\mathrm{E}_{i}\right\}_{i=0}^{d}$ (resp. $\left.\left\{\mathrm{E}_{i}^{*}\right\}_{i=0}^{d}\right)$ is an ordering of the primitive idempotents of $\mathrm{A}$ (resp. $\mathrm{A}^{*}$ ).

(LS3) $\mathrm{E}_{i}^{*} \mathrm{AE}_{j}^{*}=\mathrm{E}_{i} \mathrm{~A}^{*} \mathrm{E}_{j}=0$ if $|i-j|>1,0 \leqslant i, j \leqslant d$.

(LS4) There is no proper subspace of $W$ which is both $A$ - and $A^{*}$-invariant.

We call $d$ the diameter of $\Phi$.

We note that the above definition is taken from [13, Definition 2.1] (and the paragraph following it), and is easily shown to be equivalent to the original definition in [34, Definition 1.4].

Let $\Phi=\left(A ; A^{*} ;\left\{E_{i}\right\}_{i=0}^{d} ;\left\{E_{i}^{*}\right\}_{i=0}^{d}\right)$ be a Leonard system on W. Note that each of the following is also a Leonard system on $\mathrm{W}$ :

$$
\Phi^{*}:=\left(\mathrm{A}^{*} ; \mathrm{A} ;\left\{\mathrm{E}_{i}^{*}\right\}_{i=0}^{d} ;\left\{\mathrm{E}_{i}\right\}_{i=0}^{d}\right), \quad \Phi^{\Downarrow}:=\left(\mathrm{A} ; \mathrm{A}^{*} ;\left\{\mathrm{E}_{d-i}\right\}_{i=0}^{d} ;\left\{\mathrm{E}_{i}^{*}\right\}_{i=0}^{d}\right) .
$$

A Leonard system $\Psi$ on a vector space $W^{\prime}$ is isomorphic to $\Phi$ if there is a $\mathbb{C}$-algebra isomorphism $\sigma: \operatorname{End}(\mathrm{W}) \rightarrow \operatorname{End}\left(\mathrm{W}^{\prime}\right)$ such that $\Psi=\Phi^{\sigma}:=\left(\mathrm{A}^{\sigma} ; \mathrm{A}^{* \sigma} ;\left\{\mathrm{E}_{i}^{\sigma}\right\}_{i=0}^{d} ;\left\{\mathrm{E}_{i}^{* \sigma}\right\}_{i=0}^{d}\right)$.

For $0 \leqslant i \leqslant d$, let $\theta_{i}$ (resp. $\left.\theta_{i}^{*}\right)$ be the eigenvalue of $\mathrm{A}$ (resp. $\mathrm{A}^{*}$ ) associated with $\mathrm{E}_{i}$ (resp. $\left.\mathrm{E}_{i}^{*}\right)$. Then there are non-zero scalars $\left\{\varphi_{i}\right\}_{i=1}^{d}$ in $\mathbb{C}$ and a $\mathbb{C}$-algebra isomorphism $\sharp$ : End $(\mathrm{W}) \rightarrow$ $\operatorname{Mat}_{d+1}(\mathbb{C})$ (the full matrix algebra) such that [34, Theorem 3.2]

$$
\mathrm{A}^{\natural}=\left(\begin{array}{cccccc}
\theta_{0} & & & & & \mathbf{0} \\
1 & \theta_{1} & & & & \\
& 1 & \theta_{2} & & & \\
& & \cdot & \cdot & & \\
& & & \cdot & \cdot & \\
\mathbf{0} & & & & 1 & \theta_{d}
\end{array}\right), \quad \mathrm{A}^{* \natural}=\left(\begin{array}{cccccc}
\theta_{0}^{*} & \varphi_{1} & & & & \mathbf{0} \\
& \theta_{1}^{*} & \varphi_{2} & & & \\
& & \theta_{2}^{*} & \cdot & & \\
& & & \cdot & \cdot & \\
& & & & \cdot & \varphi_{d} \\
\mathbf{0} & & & & & \theta_{d}^{*}
\end{array}\right) .
$$

We call $\left\{\varphi_{i}\right\}_{i=1}^{d}$ the first split sequence of $\Phi$. Let $\left\{\phi_{i}\right\}_{i=1}^{d}$ be the first split sequence of $\Phi^{\Downarrow}$ and call this the second split sequence of $\Phi$. The parameter array of $\Phi$ is the sequence

$$
\left(\left\{\theta_{i}\right\}_{i=0}^{d} ;\left\{\theta_{i}^{*}\right\}_{i=0}^{d} ;\left\{\varphi_{i}\right\}_{i=1}^{d} ;\left\{\phi_{i}\right\}_{i=1}^{d}\right) .
$$

It is clear that the parameter array is a complete invariant for the isomorphism classes of Leonard systems. Terwilliger [36, Section 5] displayed all the parameter arrays of Leonard systems in parametric form. We now recall the dual $q$-Krawtchouk family of Leonard systems on which we will focus. 
Definition 5.2 ([36, Example 5.9]). The Leonard system $\Phi$ is said to have dual q-Krawtchouk type if there are scalars $\alpha, \alpha^{*}, \beta, \beta^{*}, \gamma$ with $\beta \beta^{*} \gamma \neq 0$ such that

$$
\theta_{i}=\alpha+\beta q^{-i}+\gamma q^{i}, \quad \theta_{i}^{*}=\alpha^{*}+\beta^{*} q^{-i}
$$

for $0 \leqslant i \leqslant d$, and

$$
\varphi_{i}=\beta \beta^{*} q^{1-2 i}\left(1-q^{i}\right)\left(1-q^{i-d-1}\right), \quad \phi_{i}=\gamma \beta^{*} q^{d+1-2 i}\left(1-q^{i}\right)\left(1-q^{i-d-1}\right)
$$

for $1 \leqslant i \leqslant d$. We call $\left(\alpha, \alpha^{*}, \beta, \beta^{*}, \gamma ; q, d\right)$ the parameter sequence of $\Phi$.

Take a non-zero vector $\mathrm{u} \in \mathrm{E}_{0} \mathrm{~W}$. By [35, Lemma 10.2], the vectors $\left\{\mathrm{E}_{i}^{*} \mathrm{u}\right\}_{i=0}^{d}$ form a basis for $\mathrm{W}$, called a $\Phi$-standard basis. In view of (LS3), (LS4), there are scalars $\mathrm{a}_{i}, \mathrm{~b}_{i}, \mathrm{c}_{i}, 0 \leqslant i \leqslant d$ such that $\mathrm{b}_{d}=\mathrm{c}_{0}=0, \mathrm{~b}_{i-1} \mathrm{c}_{i} \neq 0,1 \leqslant i \leqslant d$, and

$$
\text { A. } E_{i}^{*} \mathrm{u}=\mathrm{b}_{i-1} \mathrm{E}_{i-1}^{*} \mathrm{u}+\mathrm{a}_{i} \mathrm{E}_{i}^{*} \mathrm{u}+\mathrm{c}_{i+1} \mathrm{E}_{i+1}^{*} \mathrm{u}, \quad 0 \leqslant i \leqslant d,
$$

where $\mathrm{b}_{-1} \mathrm{E}_{-1}^{*} \mathrm{u}=\mathrm{c}_{d+1} \mathrm{E}_{d+1}^{*} \mathrm{u}:=0$. We call $\mathrm{a}_{i}, \mathrm{~b}_{i}, \mathrm{c}_{i}$ the intersection numbers of $\Phi$. Observe that

$$
\mathrm{a}_{i}+\mathrm{b}_{i}+\mathrm{c}_{i}=\theta_{0}, \quad 0 \leqslant i \leqslant d .
$$

By [35, Theorem 17.7], we have

$$
\begin{array}{ll}
\mathrm{b}_{i}=\varphi_{i+1} \frac{\left(\theta_{i}^{*}-\theta_{0}^{*}\right)\left(\theta_{i}^{*}-\theta_{1}^{*}\right) \cdots\left(\theta_{i}^{*}-\theta_{i-1}^{*}\right)}{\left(\theta_{i+1}^{*}-\theta_{0}^{*}\right)\left(\theta_{i+1}^{*}-\theta_{1}^{*}\right) \cdots\left(\theta_{i+1}^{*}-\theta_{i}^{*}\right)}, & 0 \leqslant i \leqslant d-1, \\
\mathrm{c}_{i}=\phi_{i} \frac{\left(\theta_{i}^{*}-\theta_{i+1}^{*}\right)\left(\theta_{i}^{*}-\theta_{i+2}^{*}\right) \cdots\left(\theta_{i}^{*}-\theta_{d}^{*}\right)}{\left(\theta_{i-1}^{*}-\theta_{i}^{*}\right)\left(\theta_{i-1}^{*}-\theta_{i+1}^{*}\right) \cdots\left(\theta_{i-1}^{*}-\theta_{d}^{*}\right)}, & 1 \leqslant i \leqslant d .
\end{array}
$$

Define a finite sequence of polynomials $\left\{\mathrm{v}_{i}\right\}_{i=0}^{d}$ in $\mathbb{C}[\xi]$ with $\operatorname{deg}\left(\mathrm{v}_{i}\right)=i$ by $\mathrm{v}_{0}:=1$ and

$$
\xi \mathrm{v}_{i}=\mathrm{b}_{i-1} \mathrm{v}_{i-1}+\mathrm{a}_{i} \mathrm{v}_{i}+\mathrm{c}_{i+1} \mathrm{v}_{i+1}, \quad 0 \leqslant i \leqslant d-1,
$$

where $b_{-1} v_{-1}:=0$. From (5.2) and (5.6) it follows that (cf. [35, Theorem 13.4])

$$
\mathrm{v}_{i}(\mathrm{~A}) \cdot \mathrm{E}_{0}^{*} \mathrm{u}=\mathrm{E}_{i}^{*} \mathrm{u}, \quad 0 \leqslant i \leqslant d .
$$

Consider the following normalization:

$$
\mathrm{f}_{i}:=\frac{\mathrm{v}_{i}}{\mathrm{v}_{i}\left(\theta_{0}\right)}, \quad 0 \leqslant i \leqslant d,
$$

where using (5.3), (5.6), we have by induction (cf. [35, Lemma 13.2])

$$
\mathrm{v}_{i}\left(\theta_{0}\right)=\frac{\mathrm{b}_{0} \mathrm{~b}_{1} \cdots \mathrm{b}_{i-1}}{\mathrm{c}_{1} \mathrm{c}_{2} \cdots \mathrm{c}_{i}}(\neq 0), \quad 0 \leqslant i \leqslant d .
$$

By [35, Theorem 17.4], for $0 \leqslant i \leqslant d$ we have

$$
\mathbf{f}_{i}=\sum_{n=0}^{i} \frac{\left(\theta_{i}^{*}-\theta_{0}^{*}\right)\left(\theta_{i}^{*}-\theta_{1}^{*}\right) \cdots\left(\theta_{i}^{*}-\theta_{n-1}^{*}\right)\left(\xi-\theta_{0}\right)\left(\xi-\theta_{1}\right) \cdots\left(\xi-\theta_{n-1}\right)}{\varphi_{1} \varphi_{2} \cdots \varphi_{n}} .
$$

Define the scalars $\mathrm{m}_{i}, 0 \leqslant i \leqslant d$, by

$$
\mathrm{m}_{i}=\operatorname{trace}\left(\mathrm{E}_{i} \mathrm{E}_{0}^{*}\right), \quad 0 \leqslant i \leqslant d,
$$


so that $\mathrm{E}_{0}^{*} \mathrm{E}_{i} \mathrm{E}_{0}^{*}=\mathrm{m}_{i} \mathrm{E}_{0}^{*}, 0 \leqslant i \leqslant d$; cf. [35, Lemma 9.2]. By [35, Theorem 17.12], we have

$$
\mathrm{m}_{i}=\frac{\varphi_{1} \varphi_{2} \cdots \varphi_{i} \phi_{1} \phi_{2} \cdots \phi_{d-i}}{\left(\theta_{0}^{*}-\theta_{1}^{*}\right) \cdots\left(\theta_{0}^{*}-\theta_{d}^{*}\right)\left(\theta_{i}-\theta_{0}\right) \cdots\left(\theta_{i}-\theta_{i-1}\right)\left(\theta_{i}-\theta_{i+1}\right) \cdots\left(\theta_{i}-\theta_{d}\right)} .
$$

Assume now that $\Phi$ has dual $q$-Krawtchouk type as in Definition 5.2. Then we have ${ }^{5}$

$$
\mathrm{b}_{i}=\beta\left(1-q^{i-d}\right), \quad \mathrm{c}_{i}=\gamma\left(1-q^{i}\right), \quad 0 \leqslant i \leqslant d .
$$

The values of the $\mathrm{f}_{i}$ at $\xi=\theta_{j}$ are given by (cf. [36, Example 5.9])

$$
\mathrm{f}_{i}\left(\theta_{j}\right)={ }_{3} \phi_{2}\left(\begin{array}{c}
q^{-i}, q^{-j}, \beta^{-1} \gamma q^{j} \\
0, q^{-d}
\end{array} \mid q, q\right), \quad 0 \leqslant i, j \leqslant d .
$$

It follows that the $\mathrm{f}_{i}$ are the dual q-Krawtchouk polynomials [14, Section 14.17] in the variable

$$
\xi=\xi(x):=\alpha+\beta q^{-x}+\gamma q^{x} .
$$

We also have

$$
\mathrm{m}_{i}=\frac{\beta^{i}}{\gamma^{i}} \frac{\left(q^{-d} ; q\right)_{i}\left(1-\beta \gamma^{-1} q^{-2 i}\right)}{q^{i(i-1)}(q ; q)_{i}\left(\beta \gamma^{-1} q^{-d-i} ; q\right)_{d+1}}, \quad 0 \leqslant i \leqslant d .
$$

Following [15], we fix a non-zero scalar $\tau$ such that

$$
\tau^{2}=\beta^{-1} \gamma,
$$

and renormalize the $\mathrm{f}_{i}$ so that they are monic ${ }^{6}$ as symmetric Laurent polynomials in $\eta$ :

$$
\mathrm{h}_{i}(\eta):=\frac{\left(q^{-d} ; q\right)_{i}}{\tau^{i}}{ }_{3} \phi_{2}\left(\begin{array}{c}
q^{-i}, \tau \eta^{-1}, \tau \eta \\
0, q^{-d}
\end{array} \mid q, q\right), \quad 0 \leqslant i \leqslant d .
$$

The $\mathrm{h}_{i}$ depend on the parameters $q, d$, and $\tau$, and we will write

$$
\mathrm{h}_{i}(\eta)=h_{i}(\eta ; \tau, d ; q), \quad 0 \leqslant i \leqslant d .
$$

We note that $\mathrm{h}_{i}$ has highest degree $i$ and lowest degree $-i$ in $\eta$, and that

$$
\mathrm{h}_{i}(\eta)=\frac{\left(q^{-d} ; q\right)_{i}}{\tau^{i}} \mathrm{f}_{i}\left(\xi\left(\log _{q} \tau^{-1} \eta\right)\right), \quad 0 \leqslant i \leqslant d .
$$

Let $\mathbf{W}$ be a vector space over $\mathbb{C}$ containing $\mathbf{W}$ as a subspace, and let $\mathbf{X} \in \operatorname{End}(\mathbf{W})$ be invertible such that $\left(\mathrm{X}+\mathrm{X}^{-1}\right) \mathrm{W} \subset \mathrm{W}$ and that

$$
\mathbf{A}=\alpha \mathbf{I}+\beta \tau\left(\mathbf{X}+\mathbf{X}^{-1}\right)=\alpha \mathbf{I}+\beta\left(\tau^{-1} \mathbf{X}\right)^{-1}+\gamma\left(\tau^{-1} \mathbf{X}\right)
$$

holds on W. Then it follows from (5.15) that

$$
\left.\mathrm{h}_{i}(\mathrm{X})\right|_{\mathrm{w}}=\frac{\left(q^{-d} ; q\right)_{i}}{\tau^{i}} \mathrm{f}_{i}(\mathrm{~A})=\tau^{i}(q ; q)_{i} \mathrm{v}_{i}(\mathrm{~A})
$$

where we have also used (5.7) and (5.9). In particular, we have

$$
\mathrm{h}_{i}(\mathrm{X}) . \mathrm{E}_{0}^{*} \mathrm{u}=\tau^{i}(q ; q)_{i} \mathrm{E}_{i}^{*} \mathrm{u}, \quad 0 \leqslant i \leqslant d .
$$

\footnotetext{
${ }^{5}$ See also [31, Theorem 2.1]. In the notation of [31, Theorem 2.1], the Leonard systems of dual $q$-Krawtchouk type correspond to Case (I) with $s^{*}=r_{1}=r_{2}=0$.

${ }^{6}$ A Laurent polynomial in $\eta$ is called monic if the coefficient of its highest degree term in $\eta$ equals 1.
} 


\section{$6 \quad$ Irreducible $T$-submodules of $\mathrm{W}$}

Recall the primary $\mathbf{T}$-module $\mathbf{W}$. In this section, we focus on the decomposition (4.3) as $T$ modules. We first discuss the primary $T$-module $M \hat{x}$. Consider the sequence

$$
\Phi:=\left.\left(A ; A^{*} ;\left\{E_{i}\right\}_{i=0}^{D} ;\left\{E_{i}^{*}\right\}_{i=0}^{D}\right)\right|_{M \hat{x}},
$$

where $\left.\right|_{M \hat{x}}$ means that each of the elements in the sequence is restricted to $M \hat{x}$.

Proposition 6.1. The sequence $\Phi$ is a Leonard system on $M \hat{x}$ that has dual q-Krawtchouk type with parameter sequence $\left(\alpha, \alpha^{*}, \beta, \beta^{*}, \gamma ; q, D\right)$, where ${ }^{7}$

$$
\begin{aligned}
& \alpha=\frac{q^{e}-1}{1-q}, \quad \beta=\frac{q^{D+e}}{q-1}, \quad \gamma=\frac{1}{1-q}, \\
& \alpha^{*}=\frac{q\left(1+q^{D+e-2}\right)}{1-q}, \quad \beta^{*}=\frac{q\left(1+q^{D+e-2}\right)\left(1+q^{D+e-1}\right)}{(q-1)\left(1+q^{e-1}\right)} .
\end{aligned}
$$

Moreover, $\left\{A_{i} \hat{x}\right\}_{i=0}^{D}$ is a $\Phi$-standard basis for $M \hat{x}$.

Proof. It follows from (2.2) and (2.3) that $\Phi$ is a Leonard system on $M \hat{x}$. Moreover, $\left\{A_{i} \hat{x}\right\}_{i=0}^{D}$ is a $\Phi$-standard basis for $M \hat{x}$, since $A_{i} \hat{x}=E_{i}^{*} \hat{X}, 0 \leqslant i \leqslant D$, and $\hat{X} \in E_{0} M \hat{x}$. By virtue of (2.4) and (5.2), we routinely obtain the parameter array of $\Phi$ from (3.1), (3.2), (3.3) using (5.4), (5.5) (or (5.9)), and find that $\Phi$ has dual $q$-Krawtchouk type as given above.

We note that the intersection numbers of $\Phi$ coincide with those of $\Gamma$.

We next consider the $T$-module $M \hat{x}^{\perp}$, the orthogonal complement of $M \hat{x}$ in $\mathbf{W}$. Observe that $E_{0} M \hat{x}^{\perp}=0$ since $E_{0} \mathbf{W}=\mathbb{C} \hat{X} \subset M \hat{x}$. From (2.1) and (3.4) it follows that

$$
\left\|E_{1} \hat{x}\right\|^{2}=\frac{\theta_{0}^{*}}{|X|}, \quad\left\langle E_{1} \hat{x}, E_{1} \hat{C}\right\rangle=\frac{1}{|X|}\left(\theta_{0}^{*}+q^{e} \theta_{1}^{*}\right), \quad \| E_{1} \hat{C}||^{2}=\frac{1+q^{e}}{|X|}\left(\theta_{0}^{*}+q^{e} \theta_{1}^{*}\right),
$$

from which it follows that

$$
w:=c \cdot\left(E_{1} \hat{C}-\frac{\theta_{0}^{*}+q^{e} \theta_{1}^{*}}{\theta_{0}^{*}} E_{1} \hat{x}\right) \in E_{1} M \hat{x}^{\perp},
$$

where we take

$$
c:=\frac{|X|\left(\alpha^{*}+\beta^{*}\right)}{\alpha^{*} \beta^{*} q^{e-1}(1-q)}
$$

for convenience. By (2.1), (3.3), (3.8), and (3.9), we routinely obtain

$$
w=\sum_{i=0}^{D-1}\left(\left(q^{-i}-1\right) \hat{C}_{i}^{-}+\left(q^{D-i-1}-1\right) \hat{C}_{i}^{+}\right) .
$$

Note that $E_{0}^{*} w=E_{D}^{*} w=0$. Let

$$
u_{i}^{\perp}:=E_{i+1}^{*} w=\left(q^{D-i-1}-1\right) \hat{C}_{i}^{+}+\left(q^{-i-1}-1\right) \hat{C}_{i+1}^{-}, \quad 0 \leqslant i \leqslant D-2 .
$$

Since $\operatorname{dim}\left(M \hat{x}^{\perp}\right)=D-1$, the vectors $\left\{u_{i}^{\perp}\right\}_{i=0}^{D-2}$ form an orthogonal basis for $M \hat{x}^{\perp}$.

Consider the sequence

$$
\Phi^{\perp}:=\left.\left(A ; A^{*} ;\left\{E_{i}\right\}_{i=1}^{D-1} ;\left\{E_{i}^{*}\right\}_{i=1}^{D-1}\right)\right|_{M \hat{x}^{\perp}} .
$$

\footnotetext{
${ }^{7}$ See also [33, Example 6.1(6)].
} 
Proposition 6.2. The T-module $M \hat{x}^{\perp}$ is irreducible, and $\Phi^{\perp}$ is a Leonard system on $M \hat{x}^{\perp}$ that has dual q-Krawtchouk type with parameter sequence $\left(\alpha^{\perp}, \alpha^{* \perp}, \beta^{\perp}, \beta^{* \perp}, \gamma^{\perp} ; q, D-2\right)$, where ${ }^{8}$

$$
\left(\alpha^{\perp}, \alpha^{* \perp}, \beta^{\perp}, \beta^{* \perp}, \gamma^{\perp}\right)=\left(\alpha, \alpha^{*}, \beta q^{-1}, \beta^{*} q^{-1}, \gamma q\right) \text {. }
$$

Moreover, $\left\{u_{i}^{\perp}\right\}_{i=0}^{D-2}$ is a $\Phi^{\perp}$-standard basis for $M \hat{x}^{\perp}$.

Proof. By Lemma 4.6, we have

$$
A \cdot u_{i}^{\perp}=\frac{q^{D+e-1}-q^{i+e}}{q-1} u_{i-1}^{\perp}+\left(q^{e}-1\right) \frac{q^{i+1}-1}{q-1} u_{i}^{\perp}+\frac{q^{i+2}-q}{q-1} u_{i+1}^{\perp}, \quad 0 \leqslant i \leqslant D-2,
$$

where $u_{-1}^{\perp}=u_{D-1}^{\perp}:=0$. Note that the coefficients of $u_{i-1}^{\perp}, u_{i+1}^{\perp}$ are non-zero. Every $T$ submodule of $M \hat{x}^{\perp}$ is spanned by some of the $u_{i}^{\perp}$ since it is $M^{*}$-invariant, and (6.3) then shows that it must be either $M \hat{x}^{\perp}$ or 0 . Hence $M \hat{x}^{\perp}$ is an irreducible $T$-module. It also follows from (6.3) that $M \hat{x}^{\perp}=M u_{0}^{\perp}$. In particular, $M \hat{x}^{\perp}$ is spanned by the vectors $E_{i} u_{0}^{\perp}, 0 \leqslant i \leqslant D$. Note that $E_{0} u_{0}^{\perp}=0$ and $E_{1} u_{0}^{\perp} \neq 0$ since $E_{0} M \hat{x}^{\perp}=0$ and $w \in E_{1} M \hat{x}^{\perp}=\mathbb{C} E_{1} u_{0}^{\perp}$. Hence it follows from (2.2) and the irreducibility of $M \hat{x}^{\perp}$ that $E_{i} u_{0}^{\perp} \neq 0,2 \leqslant i \leqslant D-1$ and $E_{D} u_{0}^{\perp}=0$. From these comments and (2.2), it follows that $\Phi^{\perp}$ is a Leonard system on $M \hat{x}^{\perp}$, and that $\left\{u_{i}^{\perp}\right\}_{i=0}^{D-2}$ is a $\Phi^{\perp}$-standard basis for $M \hat{x}^{\perp}$. Note that

$$
A^{*} . u_{i}^{\perp}=\theta_{i+1}^{*} u_{i}^{\perp}, \quad 0 \leqslant i \leqslant D-2 .
$$

The parameter array of $\Phi^{\perp}$ can now be computed as in the proof of Proposition 6.1.

We may remark that every irreducible $T$-module indeed affords a Leonard system of dual $q$-Krawtchouk type; cf. [37, Theorem 23.1].

Let $\pi$ be the orthogonal projection from $\mathbf{W}$ onto $M \hat{x}$, i.e.,

$$
(1-\pi) M \hat{x}=0, \quad \pi M \hat{x}^{\perp}=0 .
$$

Then we have $A \pi=\pi A$ and $A^{*} \pi=\pi A^{*}$ on $\mathbf{W}$. Observe that

$$
\hat{C}_{i-1}^{+}=\frac{q^{i}-1}{q^{D}-1} A_{i} \hat{x}+\frac{q^{i}}{q^{D}-1} u_{i-1}^{\perp}, \quad \hat{C}_{i}^{-}=\frac{q^{D}-q^{i}}{q^{D}-1} A_{i} \hat{x}+\frac{q^{i}}{1-q^{D}} u_{i-1}^{\perp}
$$

for $1 \leqslant i \leqslant D-1$, from which it follows that

$$
\pi \cdot \hat{C}_{i-1}^{+}=\frac{q^{i}-1}{q^{D}-1}\left(\hat{C}_{i-1}^{+}+\hat{C}_{i}^{-}\right), \quad \pi \cdot \hat{C}_{i}^{-}=\frac{q^{D}-q^{i}}{q^{D}-1}\left(\hat{C}_{i-1}^{+}+\hat{C}_{i}^{-}\right)
$$

for $1 \leqslant i \leqslant D-1$. Moreover, we have $\pi \cdot \hat{C}_{0}^{-}=\hat{C}_{0}^{-}$and $\pi \cdot \hat{C}_{D-1}^{+}=\hat{C}_{D-1}^{+}$.

\section{Irreducible $\widetilde{T}$-submodules of $\mathrm{W}$}

In this section, we focus on the decomposition (4.4) as $\widetilde{T}$-modules. We first discuss the primary $\widetilde{T}$-module $M \hat{C}$. Consider the sequence

$$
\widetilde{\Phi}:=\left.\left(A ; \widetilde{A}^{*} ;\left\{E_{i}\right\}_{i=0}^{D-1} ;\left\{\widetilde{E}_{i}^{*}\right\}_{i=0}^{D-1}\right)\right|_{M \hat{C}} .
$$

\footnotetext{
${ }^{8}$ See also [29, Theorem 6.9], [32, Theorem 4.6].
} 
Proposition 7.1. The sequence $\widetilde{\Phi}$ is a Leonard system on $M \hat{C}$ that has dual q-Krawtchouk type with parameter sequence $\left(\widetilde{\alpha}, \widetilde{\alpha}^{*}, \widetilde{\beta}, \widetilde{\beta}^{*}, \widetilde{\gamma} ; q, D-1\right)$, where ${ }^{9}$ (cf. Proposition 6.1 )

$$
\left(\widetilde{\alpha}, \widetilde{\alpha}^{*}, \widetilde{\beta}, \widetilde{\beta}^{*}, \widetilde{\gamma}\right)=\left(\alpha, \alpha^{*}, \beta, \frac{1+q^{e-1}}{1+q^{e}} \beta^{*}, \gamma\right) \text {. }
$$

Moreover, $\left\{\hat{C}_{i}\right\}_{i=0}^{D-1}$ is a $\widetilde{\Phi}$-standard basis for $M \hat{C}$.

Proof. We first remark that $E_{D} \hat{C}=0$. Indeed, the Hoffman bound $1-\theta_{0} \theta_{D}^{-1}$ on the size of a clique follows from the fact that $E_{D}$ is positive semidefinite, and the bound is attained precisely when the characteristic vector of the clique vanishes on $E_{D} V$. From this comment, (3.6), and (3.10), it follows that $\tilde{\Phi}$ is a Leonard system on $M \hat{C}$. Moreover, $\left\{\hat{C}_{i}\right\}_{i=0}^{D-1}$ is a $\widetilde{\Phi}$ standard basis for $M \hat{C}$, since $\hat{C}_{i}=\widetilde{E}_{i}^{*} \hat{X}, 0 \leqslant i \leqslant D-1$, and $\hat{X} \in E_{0} M \hat{C}$. In view of (3.11), the parameter array of $\widetilde{\Phi}$ can be computed from (3.2), (3.7), and (3.9), as in the proof of Proposition 6.1.

We note that the intersection numbers of $\widetilde{\Phi}$ coincide with those of $C$.

We next consider the $\widetilde{T}$-module $M \hat{C}^{\perp}$, the orthogonal complement of $M \hat{C}$ in $\mathbf{W}$. From (6.1) it follows that

$$
\widetilde{w}:=\widetilde{c} \cdot\left(E_{1} \hat{x}-\frac{1}{1+q^{e}} E_{1} \hat{C}\right) \in E_{1} M \hat{C}^{\perp},
$$

where we take

$$
\widetilde{c}:=\frac{|X| q\left(1+q^{e}\right)}{\beta^{*}(1-q)}
$$

for convenience. By (2.1), (3.3), (3.8), and (3.9), we obtain

$$
\widetilde{w}=\sum_{i=0}^{D-1}\left(-q^{e-i} \hat{C}_{i}^{-}+q^{-i} \hat{C}_{i}^{+}\right) .
$$

Let

$$
\widetilde{u}_{i}^{\perp}:=\widetilde{E}_{i}^{*} \widetilde{w}=-q^{e-i} \hat{C}_{i}^{-}+q^{-i} \hat{C}_{i}^{+}, \quad 0 \leqslant i \leqslant D-1 .
$$

Since $\operatorname{dim}\left(M \hat{C}^{\perp}\right)=D$, the vectors $\left\{\widetilde{u}_{i}^{\perp}\right\}_{i=0}^{D-1}$ form an orthogonal basis for $M \hat{C}^{\perp}$.

Consider the sequence

$$
\widetilde{\Phi}^{\perp}:=\left.\left(A ; \widetilde{A}^{*} ;\left\{E_{i}\right\}_{i=1}^{D} ;\left\{\widetilde{E}_{i}^{*}\right\}_{i=0}^{D-1}\right)\right|_{M \hat{C}^{\perp}} .
$$

Proposition 7.2. The $\widetilde{T}$-module $M \hat{C}^{\perp}$ is irreducible, and $\widetilde{\Phi}^{\perp}$ is a Leonard system on $M \hat{C}^{\perp}$ that has dual q-Krawtchouk type with parameter sequence $\left(\widetilde{\alpha}^{\perp}, \widetilde{\alpha}^{* \perp}, \widetilde{\beta}^{\perp}, \widetilde{\beta}^{* \perp}, \widetilde{\gamma}^{\perp} ; q, D-1\right)$, where

$$
\left(\widetilde{\alpha}^{\perp}, \widetilde{\alpha}^{* \perp}, \widetilde{\beta}^{\perp}, \widetilde{\beta}^{* \perp}, \widetilde{\gamma}^{\perp}\right)=\left(\alpha, \alpha^{*}, \beta q^{-1}, \frac{1+q^{e-1}}{1+q^{e}} \beta^{*}, \gamma q\right) .
$$

Moreover, $\left\{\widetilde{u}_{i}^{\perp}\right\}_{i=0}^{D-1}$ is a $\widetilde{\Phi}^{\perp}$-standard basis for $M \hat{C}^{\perp}$.

Proof. By Lemma 4.6, we have

$$
A . \widetilde{u}_{i}^{\perp}=\frac{q^{D+e-1}-q^{e+i-1}}{q-1} \widetilde{u}_{i-1}^{\perp}+\frac{q^{e+i}-q^{e}-q^{i+1}+1}{q-1} \widetilde{u}_{i}^{\perp}+\frac{q^{i+2}-q}{q-1} \widetilde{u}_{i+1}^{\perp}, \quad 0 \leqslant i \leqslant D-1,
$$

where $\widetilde{u}_{-1}^{\perp}=\widetilde{u}_{D}^{\perp}:=0$. The result is proved using this identity as in the proof of Proposition 6.2 .

\footnotetext{
${ }^{9}$ See [30, Proposition 4.6] for a more general result.
} 
Let $\widetilde{\pi}$ be the orthogonal projection from $\mathbf{W}$ onto $M \hat{C}$, i.e.,

$$
(1-\widetilde{\pi}) M \hat{C}=0, \quad \widetilde{\pi} M \hat{C}^{\perp}=0 .
$$

Then we have $A \widetilde{\pi}=\widetilde{\pi} A$ and $\widetilde{A}^{*} \widetilde{\pi}=\widetilde{\pi} \widetilde{A}^{*}$ on $\mathbf{W}$. Observe that

$$
\hat{C}_{i}^{-}=\frac{1}{1+q^{e}} \hat{C}_{i}-\frac{q^{i}}{1+q^{e}} \widetilde{u}_{i}^{\perp}, \quad \hat{C}_{i}^{+}=\frac{q^{e}}{1+q^{e}} \hat{C}_{i}+\frac{q^{i}}{1+q^{e}} \widetilde{u}_{i}^{\perp}
$$

for $0 \leqslant i \leqslant D-1$, from which it follows that

$$
\tilde{\pi} \cdot \hat{C}_{i}^{-}=\frac{1}{1+q^{e}}\left(\hat{C}_{i}^{-}+\hat{C}_{i}^{+}\right), \quad \tilde{\pi} \cdot \hat{C}_{i}^{+}=\frac{q^{e}}{1+q^{e}}\left(\hat{C}_{i}^{-}+\hat{C}_{i}^{+}\right)
$$

for $0 \leqslant i \leqslant D-1$.

\section{A nil-DAHA of type $\left(C_{1}^{\vee}, C_{1}\right)$}

In this section, we introduce a nil-DAHA of type $\left(C_{1}^{\vee}, C_{1}\right)$ and show that the primary T-module $\mathbf{W}$ also has a module structure for this algebra. Let $\kappa_{0}, \kappa_{1}, \kappa_{0}^{\prime}, \kappa_{1}^{\prime} \in \mathbb{C}$ be non-zero scalars. Recall that the DAHA $\mathcal{H}=\mathcal{H}\left(\kappa_{0}, \kappa_{1}, \kappa_{0}^{\prime}, \kappa_{1}^{\prime} ; q\right)$ of type $\left(C_{1}^{\vee}, C_{1}\right)$ is generated by $\mathcal{T}_{0}^{ \pm 1}, \mathcal{T}_{1}^{ \pm 1}$, and $X^{ \pm 1}$, subject to the relations ([20, Section 6.4], [25, Section 3])

$$
\left(\mathcal{T}_{i}-\kappa_{i}\right)\left(\mathcal{T}_{i}+\kappa_{i}^{-1}\right)=0, \quad\left(\mathcal{T}_{i}^{\prime}-\kappa_{i}^{\prime}\right)\left(\mathcal{T}_{i}^{\prime}+\kappa_{i}^{\prime-1}\right)=0, \quad i=0,1,
$$

where

$$
\mathcal{T}_{0}^{\prime}:=q^{-1 / 2} X \mathcal{T}_{0}^{-1}, \quad \mathcal{T}_{1}^{\prime}:=X^{-1} \mathcal{T}_{1}^{-1}
$$

Cherednik and Orr [9] (cf. [7, 8]) introduced the concept of nil-DAHAs for reduced affine root systems. The procedure for obtaining nil-DAHAs from ordinary DAHAs discussed in [9, Section 2.5] works for the non-reduced affine root systems of type $\left(C_{n}^{\vee}, C_{n}\right)$ as well, with a bit of extra flexibility in the specialization. ${ }^{10}$ It will turn out that the following specialization for type $\left(C_{1}^{\vee}, C_{1}\right)$ is the one which is well-suited to our situation:

Definition 8.1. Let $\kappa, \kappa^{\prime} \in \mathbb{C}$ be non-zero scalars. Let $\overline{\mathcal{H}}=\overline{\mathcal{H}}\left(\kappa, \kappa^{\prime}\right)$ be the $\mathbb{C}$-algebra generated by $\mathcal{T}^{ \pm 1}, \mathcal{U}$, and $X^{ \pm 1}$, subject to the relations ${ }^{11}$

$$
(\mathcal{T}-\kappa)\left(\mathcal{T}+\kappa^{-1}\right)=0, \quad\left(\mathcal{T}^{\prime}-\kappa^{\prime}\right)\left(\mathcal{T}^{\prime}+\kappa^{\prime-1}\right)=0, \quad \mathcal{U}(\mathcal{U}+1)=0, \quad \mathcal{U}^{\prime 2}=0,
$$

where

$$
\mathcal{T}^{\prime}=X \mathcal{T}^{-1}, \quad \mathfrak{U}^{\prime}=X^{-1}(\mathcal{U}+1) .
$$

We call $\overline{\mathcal{H}}$ a nil-DAHA of type $\left(C_{1}^{\vee}, C_{1}\right)$.

Remark 8.2. Our nil-DAHA $\overline{\mathcal{H}}$ is obtained from $\mathcal{H}$ as follows. Let $\ddot{\mathcal{T}}_{1}:=\kappa_{1} \mathcal{T}_{1}, \ddot{\mathfrak{T}}_{1}^{\prime}:=\kappa_{1} \mathcal{T}_{1}^{\prime}$. Then

$$
\ddot{\mathcal{T}}_{1}^{\prime}=\kappa_{1} \mathcal{X}^{-1}\left(\mathcal{T}_{1}-\kappa_{1}+\kappa_{1}^{-1}\right)=X^{-1}\left(\ddot{\mathcal{T}}_{1}-\kappa_{1}^{2}+1\right),
$$

\footnotetext{
${ }^{10}$ We learned this procedure for type $\left(C_{n}^{\vee}, C_{n}\right)$ from Daniel Orr.

${ }^{11}$ The definition of a nil-DAHA of type $\left(C_{1}^{\vee}, C_{1}\right)$ given here is different from the one in [18, Definition 5.1]. In fact, the former is a homomorphic image of the latter.
} 
and we have

$$
\left(\ddot{\mathcal{T}}_{1}-\kappa_{1}^{2}\right)\left(\ddot{\mathfrak{T}}_{1}+1\right)=0, \quad\left(\ddot{\mathcal{T}}_{1}^{\prime}-\kappa_{1} \kappa_{1}^{\prime}\right)\left(\ddot{\mathcal{T}}_{1}^{\prime}+\kappa_{1} \kappa_{1}^{\prime-1}\right)=0 .
$$

Take $\mathcal{T}_{0}^{ \pm 1}, X^{ \pm 1}$, and $\ddot{\mathcal{T}}_{1}$ as new generators for $\mathcal{H}$. We now set $\kappa_{1}^{\prime}=1$ and let $\kappa_{1} \rightarrow 0$. Then (8.1) and (8.2) become

$$
\ddot{\mathcal{T}}_{1}^{\prime}=X^{-1}\left(\ddot{\mathfrak{T}}_{1}+1\right), \quad \ddot{\mathcal{T}}_{1}\left(\ddot{\mathfrak{T}}_{1}+1\right)=0, \quad\left(\ddot{\mathfrak{T}}_{1}^{\prime}\right)^{2}=0,
$$

and we obtain the presentation of $\overline{\mathcal{H}}$ in Definition 8.1 by the replacement

$$
\left(\mathcal{T}_{0}, q^{-1 / 2} X, \ddot{\mathcal{T}}_{1}, \kappa_{0}, \kappa_{0}^{\prime}\right) \rightarrow\left(\mathcal{T}, X, \mathcal{U}, \kappa, \kappa^{\prime}\right) .
$$

We note that, if we instead set $\kappa_{1}^{\prime}=\kappa_{1}$ and let $\kappa_{1} \rightarrow 0$, then the second identity in (8.2) becomes

$$
\ddot{\mathcal{T}}_{1}^{\prime}\left(\ddot{\mathcal{T}}_{1}^{\prime}+1\right)=0 .
$$

This gives another version of a nil-DAHA of type $\left(C_{1}^{\vee}, C_{1}\right)$, which we expect would be suitable for the Grassmann graphs [2, Section 9.3] corresponding to the dual $q$-Hahn polynomials [14, Section 14.7]. We may also apply the above procedure to $\mathcal{T}_{0}$ as well to get more variations.

Remark 8.3. By either or both of the replacements $\mathcal{T} \rightarrow-\mathcal{T}, X \rightarrow-X$, it is immediate to see that the four nil-DAHAs $\overline{\mathcal{H}}\left( \pm \kappa, \pm \kappa^{\prime}\right)$ are all isomorphic.

Remark 8.4. Mazzocco [22] defined seven new algebras as confluences of the DAHA $\mathcal{H}$, and established a new link between the theory of the Painlevé equations and part of the left side of the $q$-Askey scheme. One of these algebras, called $\mathcal{H}_{\mathrm{III}}$, turns out to be isomorphic to the $\mathbb{C}$ algebra having the same generators and relations as the nil-DAHA $\overline{\mathcal{H}}$, plus one further relation $\mathcal{U}^{\prime}=q \mathcal{U}$; cf. [22, equations (3.86)-(3.91)].

For the rest of this paper, we set

$$
\kappa=q^{-e / 2}, \quad \kappa^{\prime}=\sqrt{-1} q^{-D / 2}
$$

in Definition 8.1, where we recall that $q$ is assumed to be a prime power in our context. Our first goal is to define a $2 D$-dimensional representation of $\overline{\mathcal{H}}=\overline{\mathcal{H}}\left(\kappa, \kappa^{\prime}\right)$. To this end, we consider the following matrices:

- for $0 \leqslant i \leqslant D-1$, let

$$
t(i)=\left(\begin{array}{cc}
q^{-e / 2}-q^{e / 2} & q^{e / 2} \\
q^{-e / 2} & 0
\end{array}\right), \quad u^{\prime}(i)=\left(\begin{array}{cc}
0 & 0 \\
-\sqrt{-1} q^{(D-e) / 2-i} & 0
\end{array}\right)
$$

- for $1 \leqslant i \leqslant D-1$, let

$$
t^{\prime}(i)=\sqrt{-1}\left(\begin{array}{cc}
q^{-D / 2}\left(q^{D}-q^{i}+1\right) & q^{D / 2}\left(q^{i-D}-1\right) \\
q^{-D / 2}\left(1-q^{i}\right) & q^{i-D / 2}
\end{array}\right), \quad u(i)=\left(\begin{array}{cc}
-1 & 1-q^{D-i} \\
0 & 0
\end{array}\right)
$$

and let $t^{\prime}(0)=\left(\sqrt{-1} q^{-D / 2}\right), t^{\prime}(D)=\left(\sqrt{-1} q^{-D / 2}\right), u(0)=(0)$, and $u(D)=(-1)$.

Lemma 8.5. The following $(i)$, (ii) hold:

(i) $(t(i)-\kappa)\left(t(i)+\kappa^{-1}\right)=0$ for $0 \leqslant i \leqslant D-1$.

(ii) $\left(t^{\prime}(i)-\kappa^{\prime}\right)\left(t^{\prime}(i)+\kappa^{\prime-1}\right)=0$ for $0 \leqslant i \leqslant D$. 
Proof. (i) Immediate from $\operatorname{det}(t(i))=-1$ and trace $(t(i))=\kappa-\kappa^{-1}$.

(ii) Similar to the proof of (i) above.

Lemma 8.6. The following $(i)$, (ii) hold:

(i) $u(i)(u(i)+1)=0$ for $0 \leqslant i \leqslant D$.

(ii) $\left(u^{\prime}(i)\right)^{2}=0$ for $0 \leqslant i \leqslant D-1$.

Proof. Routine.

Define the $2 D \times 2 D$ block diagonal matrices $\mathfrak{t}, \mathfrak{t}^{\prime}, \mathfrak{u}$, and $\mathfrak{u}^{\prime}$ by

$$
\begin{aligned}
& \mathfrak{t}=\operatorname{blockdiag}(t(0), t(1), \ldots, t(D-1)), \\
& \mathfrak{t}^{\prime}=\operatorname{blockdiag}\left(t^{\prime}(0), t^{\prime}(1), \ldots, t^{\prime}(D-1), t^{\prime}(D)\right), \\
& \mathfrak{u}=\operatorname{blockdiag}(u(0), u(1), \ldots, u(D-1), u(D)), \\
& \mathfrak{u}^{\prime}=\operatorname{blockdiag}\left(u^{\prime}(0), u^{\prime}(1), \ldots, u^{\prime}(D-1)\right) .
\end{aligned}
$$

Moreover, let

$$
\mathfrak{x}=\mathfrak{t}^{\prime} \mathfrak{t} .
$$

Proposition 8.7. There is a representation $\overline{\mathcal{H}} \rightarrow \operatorname{Mat}_{2 D}(\mathbb{C})$ such that

$$
\mathcal{T} \mapsto \mathfrak{t}, \quad \mathcal{T}^{\prime} \mapsto \mathfrak{t}^{\prime}, \quad \mathcal{U} \mapsto \mathfrak{u}, \quad \mathcal{U}^{\prime} \mapsto \mathfrak{u}^{\prime}, \quad X \mapsto \mathfrak{x} .
$$

Proof. From Lemmas 8.5 and 8.6 it follows that

$$
(\mathfrak{t}-\kappa)\left(\mathfrak{t}+\kappa^{-1}\right)=\left(\mathfrak{t}^{\prime}-\kappa^{\prime}\right)\left(\mathfrak{t}^{\prime}+\kappa^{\prime-1}\right)=\mathfrak{u}(\mathfrak{u}+1)=\mathfrak{u}^{\prime 2}=0 .
$$

In particular, $\mathfrak{t}, \mathfrak{t}^{\prime}$, and $\mathfrak{x}$ are invertible. We have $\mathfrak{t}^{\prime}=\mathfrak{x t}^{-1}$ by definition, and it is a straightforward matter to show that $\mathfrak{x} \mathfrak{u}^{\prime}=\mathfrak{u}+1$. It follows that $\mathfrak{t}^{ \pm 1}, \mathfrak{u}$, and $\mathfrak{x}^{ \pm 1}$ satisfy the defining relations for $\overline{\mathcal{H}}$, and the result follows.

Corollary 8.8. There is an $\overline{\mathcal{H}}$-module structure on $\mathbf{W}$ such that $\mathfrak{t}, \mathfrak{t}^{\prime}, \mathfrak{u}, \mathfrak{u}^{\prime}$, and $\mathfrak{x}$ are respectively the matrices representing the actions of $\mathcal{T}, \mathcal{T}^{\prime}, \mathcal{U}, \mathcal{U}^{\prime}$, and $\mathcal{X}$ in the ordered basis $\mathcal{C}$ from (4.2).

By Corollary 8.8, $\mathbf{W}$ is now a module for both $\mathbf{T}$ and $\overline{\mathcal{H}}=\overline{\mathcal{H}}\left(\kappa, \kappa^{\prime}\right)$, where $\kappa, \kappa^{\prime}$ are given as in (8.3). We next discuss how the two module structures are related. Let

$$
\mathcal{A}=\mathcal{X}+\mathfrak{X}^{-1}, \quad \mathcal{A}^{*}=\sqrt{-1} q^{-D / 2}\left(\mathcal{T U}^{\prime}+\mathcal{U} \mathcal{T}^{\prime}\right), \quad \tilde{\mathcal{A}}^{*}=\sqrt{-1} q^{-D / 2}\left(\mathcal{T} \mathcal{U}^{\prime}+q \mathcal{U} \mathcal{T}^{\prime}\right) .
$$

For the rest of this paper, we also let

$$
\tau=\sqrt{-1} q^{-(D+e) / 2} .
$$

Note that $\tau^{2}=\beta^{-1} \gamma$, where $\beta, \gamma$ are from Proposition 6.1; cf. (5.12).

The following four lemmas are checked by straightforward calculations. Recall (4.1).

Lemma 8.9. For $0 \leqslant i \leqslant D-1$, the actions of $X^{ \pm 1}$ on $\hat{C}_{i}^{-}$are given as linear combinations with the following terms and coefficients:

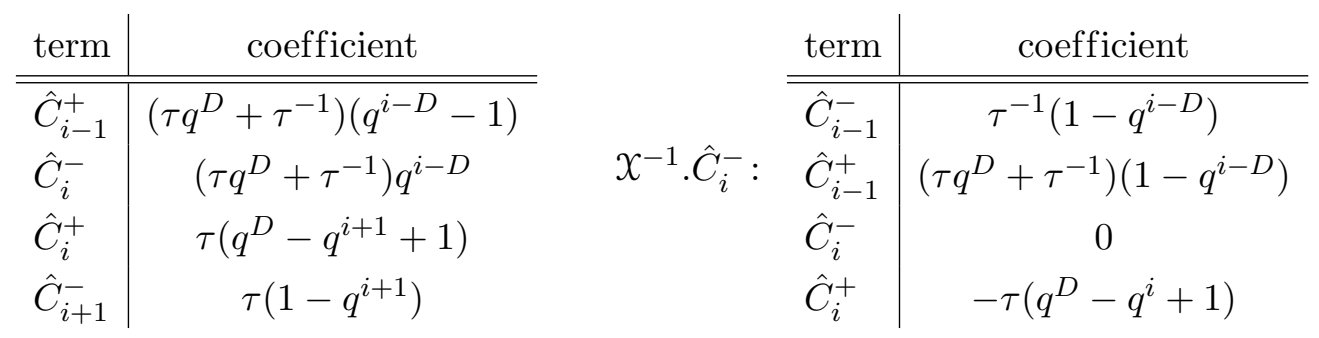


Lemma 8.10. For $0 \leqslant i \leqslant D-1$, the actions of $X^{ \pm 1}$ on $\hat{C}_{i}^{+}$are given as linear combinations with the following terms and coefficients:

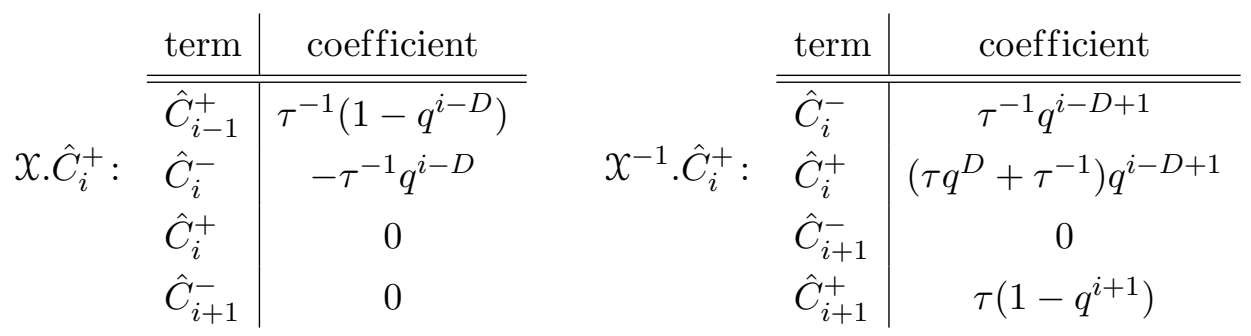

Lemma 8.11. For $0 \leqslant i \leqslant D-1$, the actions of $\mathcal{A}$ on $\hat{C}_{i}^{ \pm}$are given as linear combinations with the following terms and coefficients:

\begin{tabular}{|c|c|c|c|c|}
\hline term & coefficient & & term & coefficient \\
\hline$\hat{C}_{i-1}^{-}$ & $\tau^{-1}\left(1-q^{i-D}\right)$ & & $\hat{C}_{i-1}^{+}$ & $\tau^{-1}\left(1-q^{i-D}\right)$ \\
\hline$\hat{C}_{i-1}^{+}$ & 0 & & $\hat{C}_{i}^{-}$ & $\tau^{-1} q^{i-D}(q-1)$ \\
\hline$\hat{C}_{i}^{-}$ & $\left(\tau q^{D}+\tau^{-1}\right) q^{i-D}$ & & $\hat{C}_{i}^{+}$ & $\left(\tau q^{D}+\tau^{-1}\right) q^{i-D+1}$ \\
\hline$\hat{C}_{i}^{+}$ & $\tau q^{i}(1-q)$ & & $\hat{C}_{i+1}^{-}$ & 0 \\
\hline$\hat{C}_{i+1}^{-}$ & $\tau\left(1-q^{i+1}\right)$ & & $\hat{C}_{i+1}^{+}$ & $\tau\left(1-q^{i+1}\right)$ \\
\hline
\end{tabular}

Lemma 8.12. For $0 \leqslant i \leqslant D-1$, the actions of $\mathcal{A}^{*}, \widetilde{\mathcal{A}}^{*}$ on $\hat{C}_{i}^{ \pm}$are given by

$$
\begin{array}{ll}
\mathcal{A}^{*} . \hat{C}_{i}^{-}=q^{-i} \hat{C}_{i}^{-}, & \mathcal{A}^{*} . \hat{C}_{i}^{+}=q^{-i-1} \hat{C}_{i}^{+}, \\
\widetilde{\mathcal{A}}^{*} . \hat{C}_{i}^{-}=q^{-i} \hat{C}_{i}^{-}, & \widetilde{\mathcal{A}}^{*} . \hat{C}_{i}^{+}=q^{-i} \hat{C}_{i}^{+} .
\end{array}
$$

Recall the generators $A, A^{*}$, and $\widetilde{A}^{*}$ of $\mathbf{T}$. We now present our first main result.

Theorem 8.13. On $\mathbf{W}$, we have

$$
A=\alpha+\beta \tau \mathcal{A}, \quad A^{*}=\alpha^{*}+\beta^{*} \mathcal{A}^{*}, \quad \widetilde{A}^{*}=\widetilde{\alpha}^{*}+\widetilde{\beta}^{*} \widetilde{\mathcal{A}}^{*},
$$

where $\alpha, \alpha^{*}, \widetilde{\alpha}^{*}, \beta, \beta^{*}, \widetilde{\beta}^{*}$ are from Propositions 6.1 and 7.1. Moreover, $\mathbf{W}$ is an irreducible $\overline{\mathcal{H}}$-module.

Proof. The three identities follow from Lemmas 4.6, 4.7, 8.11, 8.12, and Propositions 6.1, 7.1. Since $\mathbf{W}$ is an irreducible $\mathbf{T}$-module by Proposition 4.5 and since $A, A^{*}$, and $\widetilde{A}^{*}$ generate $\mathbf{T}$, it follows from these identities that $\mathbf{W}$ is irreducible as an $\overline{\mathcal{H}}$-module.

Recall the orthogonal projection $\pi$ (resp. $\tilde{\pi}$ ) from $\mathbf{W}$ onto $M \hat{x}$ (resp. $M \hat{C}$ ). The following result illustrates (to some extent) how we arrived at the $\overline{\mathcal{H}}$-module structure on $\mathbf{W}$ given above:

Theorem 8.14. On $\mathbf{W}$, we have

$$
\pi=\frac{\mathcal{T}^{\prime}+\kappa^{\prime-1}}{\kappa^{\prime}+\kappa^{\prime-1}}, \quad \tilde{\pi}=\frac{\mathcal{T}+\kappa^{-1}}{\kappa+\kappa^{-1}} .
$$

Proof. Use (6.5), (7.3).

Remark 8.15. A direct computation shows that we also have $\mathfrak{u}^{\prime}=q \mathfrak{u x}$ in Proposition 8.7, so that, by Theorem $8.13, \mathbf{W}$ is indeed an irreducible module for the algebra $\mathcal{H}_{\text {III }}$ mentioned in Remark 8.4. The finite-dimensional irreducible modules for $\mathcal{H}$ have been classified by Oblomkov 
and Stoica [24] in the general case where the scalar $q$ is not a root of unity. See also [23]. It would be an interesting problem to specialize the classification to $\overline{\mathcal{H}}$ and/or $\mathcal{H}_{\mathrm{III}}$. We may remark that Mazzocco [21, Section 3] obtained (among other results) a faithful representation of $\mathcal{H}_{\text {III }}$ on $\mathbb{C}\left[\eta, \eta^{-1}\right]$. She introduced the non-symmetric Al-Salam-Chihara polynomials in proving the faithfulness of this representation, and the non-symmetric dual $q$-Krawtchouk polynomials $\ell_{i}^{ \pm}$ which we will discuss in Sections 9 and 10 are a discretization of these Laurent polynomials.

\section{Non-symmetric dual $q$-Krawtchouk polynomials}

Recall the four Leonard systems $\Phi, \Phi^{\perp}, \widetilde{\Phi}$, and $\widetilde{\Phi}^{\perp}$, and their standard bases $\left\{A_{i} \hat{x}\right\}_{i=0}^{D},\left\{u_{i}^{\perp}\right\}_{i=0}^{D-2}$, $\left\{\hat{C}_{i}\right\}_{i=0}^{D-1}$, and $\left\{\widetilde{u}_{i}^{\perp}\right\}_{i=0}^{D-1}$ from Propositions 6.1, 6.2, 7.1, and 7.2, respectively. We consider the monic dual $q$-Krawtchouk (Laurent) polynomials from (5.13) attached to these Leonard systems. More specifically, with the notation (5.14) we let

$$
\begin{array}{ll}
h_{i}(\eta)=h_{i}(\eta ; \tau, D ; q), & 0 \leqslant i \leqslant D, \\
h_{i}^{\perp}(\eta)=h_{i}(\eta ; \tau q, D-2 ; q), & 0 \leqslant i \leqslant D-2, \\
\widetilde{h}_{i}(\eta)=h_{i}(\eta ; \tau, D-1 ; q), & 0 \leqslant i \leqslant D-1, \\
\widetilde{h}_{i}^{\perp}(\eta)=h_{i}(\eta ; \tau q, D-1 ; q), & 0 \leqslant i \leqslant D-1
\end{array}
$$

for $\Phi, \Phi^{\perp}, \widetilde{\Phi}$, and $\widetilde{\Phi}^{\perp}$, respectively, where the scalar $\tau$ is from (8.4). Indeed, we have $\beta^{-1} \gamma=$ $\widetilde{\beta}^{-1} \widetilde{\gamma}=\tau^{2}$ and $\left(\beta^{\perp}\right)^{-1} \gamma^{\perp}=\left(\widetilde{\beta}^{\perp}\right)^{-1} \widetilde{\gamma}^{\perp}=(\tau q)^{2}$. We moreover set

$$
\begin{aligned}
& p^{\perp}(\eta)=\eta^{-1}(\eta-\tau)\left(\eta-\tau^{-1} q^{-D}\right), \\
& \widetilde{p}(\eta)=\eta^{-1}\left(\eta-\tau^{-1} q^{-D}\right), \\
& \widetilde{p}^{\perp}(\eta)=\eta^{-1}(\eta-\tau) .
\end{aligned}
$$

Note that $p^{\perp}, \widetilde{p}$, and $\widetilde{p}^{\perp}$ are monic. Using these Laurent polynomials, we now define

$$
\begin{aligned}
\ell_{i}^{+}(\eta) & =\frac{h_{i+1}-p^{\perp} h_{i}^{\perp}}{\tau^{i+1}\left(1-q^{D}\right)(q ; q)_{i}}, & \ell_{i}^{-}(\eta) & =\frac{q^{D}-q^{i}}{\tau^{i}\left(q^{D}-1\right)(q ; q)_{i}}\left(h_{i}-\frac{1-q^{i}}{q^{D}-q^{i}} p^{\perp} h_{i-1}^{\perp}\right), \\
\tilde{\ell}_{i}^{+}(\eta) & =\frac{q^{e}\left(\widetilde{p} \widetilde{h}_{i}-\widetilde{p}^{\perp} \widetilde{h}_{i}^{\perp}\right)}{\tau^{i}\left(1+q^{e}\right)(q ; q)_{i}}, & \tilde{\ell}_{i}^{-}(\eta) & =\frac{\widetilde{p} \widetilde{h}_{i}+q^{e} \widetilde{p}^{\perp} \widetilde{h}_{i}^{\perp}}{\tau^{i}\left(1+q^{e}\right)(q ; q)_{i}}
\end{aligned}
$$

for $0 \leqslant i \leqslant D-1$, where $h_{-1}^{\perp}:=0$ and

$$
h_{D-1}^{\perp}(\eta):=\prod_{n=1}^{D-1}\left(\eta+\eta^{-1}-\tau q^{n}-\tau^{-1} q^{-n}\right)=\eta^{1-D} \prod_{n=1}^{D-1}\left(\eta-\tau q^{n}\right)\left(\eta-\tau^{-1} q^{-n}\right) .
$$

Let

$$
\mathfrak{L}=\sum_{i=-D}^{D-1} \mathbb{C} \eta^{i} \subset \mathbb{C}\left[\eta, \eta^{-1}\right]
$$

Observe that $\operatorname{dim}(\mathfrak{L})=2 D$, and that the $\ell_{i}^{ \pm}$and the $\widetilde{\ell}_{i}^{ \pm}$all belong to $\mathfrak{L}$. Our definition of these Laurent polynomials is explained by the following result (and its proof):

Proposition 9.1. For $0 \leqslant i \leqslant D-1$, we have

$$
\hat{C}_{i}^{+}=\ell_{i}^{+}(X) \cdot \hat{x}=\widetilde{\ell}_{i}^{+}(X) \cdot \hat{x}, \quad \hat{C}_{i}^{-}=\ell_{i}^{-}(X) \cdot \hat{x}=\tilde{\ell}_{i}^{-}(X) \cdot \hat{x} .
$$

In particular, we have $\ell_{i}^{+}=\widetilde{\ell}_{i}^{+}, \ell_{i}^{-}=\tilde{\ell}_{i}^{-}$for $0 \leqslant i \leqslant D-1$. Moreover, the $\ell_{i}^{ \pm}$form a basis for $\mathfrak{L}$. 
Proof. Setting $i=0$ in Lemma 8.9, we have

$$
\text { X. } \hat{x}=\left(\tau+\tau^{-1} q^{-D}\right) \hat{x}+\tau\left(q^{D}-q+1\right) \hat{C}_{0}^{+}+\tau(1-q) \hat{C}_{1}^{-}, \quad X^{-1} . \hat{x}=-\tau q^{D} \hat{C}_{0}^{+} .
$$

By these identities, (6.2), and (7.1), we have

$$
u_{0}^{\perp}=\tau^{-1} q^{-1} p^{\perp}(\mathcal{X}) . \hat{x}, \quad \hat{C}_{0}=\widetilde{p}(\mathcal{X}) . \hat{x}, \quad \widetilde{u}_{0}^{\perp}=-q^{e} \widetilde{p}^{\perp}(X) . \hat{x} .
$$

We first show that $\hat{C}_{i}^{+}=\ell_{i}^{+}(X) . \hat{x}$. By virtue of Propositions 6.1, 6.2, and Theorem 8.13, we may apply the discussions in the last paragraph of Section 5 to the Leonard systems $\Phi, \Phi^{\perp}$. Assume that $0 \leqslant i \leqslant D-2$. Then, from (5.16) and (6.4) it follows that

$$
\hat{C}_{i}^{+}=\frac{q^{i+1}-1}{q^{D}-1} \frac{h_{i+1}(X)}{\tau^{i+1}(q ; q)_{i+1}} \cdot \hat{x}+\frac{q^{i+1}}{q^{D}-1} \frac{h_{i}^{\perp}(X)}{\tau^{i} q^{i}(q ; q)_{i}} \cdot u_{0}^{\perp},
$$

which, together with (9.3), gives $\hat{C}_{i}^{+}=\ell_{i}^{+}(\mathcal{X}) . \hat{x}$. When $i=D-1$, by (5.16) we have

$$
\hat{C}_{D-1}^{+}=A_{D} \hat{x}=\frac{h_{D}(X)}{\tau^{D}(q ; q)_{D}} . \hat{x} .
$$

On the other hand, from Proposition 6.1 and Theorem 8.13, it follows that

$$
h_{D-1}^{\perp}(X)=\beta^{1-D} \tau^{1-D} \prod_{n=1}^{D-1}\left(A-\theta_{n}\right)
$$

on W. Hence it follows from Proposition 6.2 that $h_{D-1}^{\perp}(X)$ vanishes on $M \hat{x}^{\perp}$, so that by (9.3) we have in particular

$$
h_{D-1}^{\perp}(X) p^{\perp}(X) \cdot \hat{x}=\tau q h_{D-1}^{\perp}(X) \cdot u_{0}^{\perp}=0 .
$$

Combining these comments, we obtain $\hat{C}_{D-1}^{+}=\ell_{D-1}^{+}(\mathcal{X}) . \hat{x}$. The other identities in (9.2) are similarly (and more easily) proved, using also Propositions 7.1, 7.2, and (7.2).

Finally, from (9.2) it follows that the $\ell_{i}^{ \pm}$and the $\widetilde{\ell}_{i}^{ \pm}$form two bases for $\mathfrak{L}$, but then (9.2) again shows that we must have $\ell_{i}^{+}=\widetilde{\ell}_{i}^{+}, \ell_{i}^{-}=\widetilde{\ell}_{i}^{-}$for $0 \leqslant i \leqslant D-1$. This completes the proof.

Definition 9.2. We call the Laurent polynomials $\ell_{i}^{ \pm}=\widetilde{\ell}_{i}^{ \pm}, 0 \leqslant i \leqslant D-1$, the non-symmetric dual q-Krawtchouk polynomials.

Remark 9.3. We have given two expressions for the non-symmetric dual $q$-Krawtchouk polynomials. The first one, i.e., the $\ell_{i}^{ \pm}$, is a specialization of the expressions for the non-symmetric $q$-Racah polynomials ${ }^{12}$ in [17, Proposition 7.8] and for the non-symmetric Askey-Wilson polynomials in [15, Section 4] (but with a different normalization; cf. [17, Section 11]). It is a natural guess that the polynomials studied in $[15,17]$ also have expressions akin to the second one, i.e., the $\widetilde{\ell}_{i}^{ \pm}$.

We next show that the $\ell_{i}^{ \pm}$satisfy recurrence relations with at most four terms. As a byproduct of the proof of Proposition 9.1 it follows that

Lemma 9.4. On $\mathbf{W}$, we have

$$
p^{\perp}(X) h_{D-1}^{\perp}(X)=0 .
$$

\footnotetext{
${ }^{12}$ Strictly speaking, this is true except for $\ell_{D-1}^{+}$, in which case we adjusted it so that it has no term of degree $D$. A similar adjustment should also be possible for the non-symmetric $q$-Racah polynomials.
} 
Proof. From Proposition 9.1 and (9.4) it follows that

$$
p^{\perp}(X) h_{D-1}^{\perp}(X) \cdot \hat{C}_{i}^{ \pm}=\ell_{i}^{ \pm}(X) h_{D-1}^{\perp}(X) p^{\perp}(X) \cdot \hat{x}=0
$$

for $0 \leqslant i \leqslant D-1$.

For convenience, let

$$
\ell_{-1}^{ \pm}(\eta):=0, \quad \ell_{D}^{+}(\eta):=-\frac{\eta^{-1} p^{\perp} h_{D-1}^{\perp}}{\tau^{D+1}(q ; q)_{D}}, \quad \ell_{D}^{-}(\eta):=\frac{p^{\perp} h_{D-1}^{\perp}}{\tau^{D}(q ; q)_{D}}
$$

Observe that

$$
\eta^{-1} p^{\perp} h_{D-1}^{\perp} \equiv q^{-D} \eta^{-D-1} \quad(\bmod \mathfrak{L}), \quad p^{\perp} h_{D-1}^{\perp} \equiv \eta^{D} \quad(\bmod \mathfrak{L}) .
$$

Theorem 9.5. The following $(i)$, (ii) hold:

(i) For $0 \leqslant i \leqslant D-1, \eta^{ \pm 1} \ell_{i}^{-}$are linear combinations with the following terms and coefficients:

$$
\begin{array}{l|ccc|c}
\text { term } & \text { coefficient } & & \text { term } & \text { coefficient } \\
\hline \hline \ell_{i-1}^{+} & \left(\tau q^{D}+\tau^{-1}\right)\left(q^{i-D}-1\right) & & \ell_{i-1}^{-} & \tau^{-1}\left(1-q^{i-D}\right) \\
\ell_{i}^{-} & \left(\tau q^{D}+\tau^{-1}\right) q^{i-D} & \eta^{-1} \ell_{i}^{-}: & \ell_{i-1}^{+} & \left(\tau q^{D}+\tau^{-1}\right)\left(1-q^{i-D}\right) \\
\ell_{i}^{+} & \tau\left(q^{D}-q^{i+1}+1\right) & & \ell_{i}^{-} & 0 \\
\ell_{i+1}^{-} & \tau\left(1-q^{i+1}\right) & & \ell_{i}^{+} & -\tau\left(q^{D}-q^{i}+1\right)
\end{array}
$$

(ii) For $0 \leqslant i \leqslant D-1, \eta^{ \pm 1} \ell_{i}^{+}$are linear combinations with the following terms and coefficients:

\begin{tabular}{rc|ccc|c} 
& term & coefficient & & term & coefficient \\
\hline \hline$\ell_{i-1}^{+}$ & $\tau^{-1}\left(1-q^{i-D}\right)$ & & $\ell_{i}^{-}$ & $\tau^{-1} q^{i-D+1}$ \\
$\eta \ell_{i}^{+}:$ & $\ell_{i}^{-}$ & $-\tau^{-1} q^{i-D}$ & $\eta^{-1} \ell_{i}^{+}:$ & $\ell_{i}^{+}$ & $\left(\tau q^{D}+\tau^{-1}\right) q^{i-D+1}$ \\
$\ell_{i}^{+}$ & 0 & & $\ell_{i+1}^{-}$ & 0 \\
$\ell_{i+1}^{-}$ & 0 & & $\ell_{i+1}^{+}$ & $\tau\left(1-q^{i+1}\right)$
\end{tabular}

Proof. Looking at the two expressions $\ell_{i}^{ \pm}=\tilde{\ell}_{i}^{ \pm}$it follows that $\ell_{i}^{+}$has highest degree (at most) $i-1$ and lowest degree $-i-1$, whereas $\ell_{i}^{-}$has highest degree $i$ and lowest degree $-i$. Hence, except for $\eta^{-1} \ell_{D-1}^{+}, \eta \ell_{D-1}^{-}$we have $\eta^{ \pm 1} \ell_{i}^{ \pm} \in \mathfrak{L}$, and the result follows from Lemmas 8.9, 8.10 and Proposition 9.1. For the remaining two cases, using (9.5) we routinely find that

$$
\eta^{-1} \ell_{D-1}^{+} \equiv \tau\left(1-q^{D}\right) \ell_{D}^{+}(\bmod \mathfrak{L}), \quad \eta \ell_{D-1}^{-} \equiv \tau\left(1-q^{D}\right) \ell_{D}^{-} \quad(\bmod \mathfrak{L}) .
$$

Since $\ell_{D}^{ \pm}(\mathcal{X})$ vanish on $\mathbf{W}$ by virtue of Lemma 9.4 , the result again follows from Lemmas 8.9, 8.10 and Proposition 9.1.

\section{Orthogonality relations}

Recall the subspace $\mathfrak{L}$ of $\mathbb{C}\left[\eta, \eta^{-1}\right]$ from (9.1). In this section, we define a Hermitian inner product on $\mathfrak{L}$ and show that the non-symmetric dual $q$-Krawtchouk polynomials $\ell_{i}^{ \pm}$are orthogonal with respect to that inner product. 
From Proposition 9.1 and Lemma 9.4 it follows that the minimal polynomial of $\mathcal{X}$ on $\mathbf{W}$ has degree $2 D$ and is given by $\eta^{D} p^{\perp} h_{D-1}^{\perp}$, which has the following $2 D$ simple zeros:

$$
\lambda_{i}:=\tau q^{i}, \quad 0 \leqslant i \leqslant D-1, \quad \lambda_{-i}:=\tau^{-1} q^{-i}, \quad 1 \leqslant i \leqslant D .
$$

In particular, $\mathcal{X}$ is multiplicity-free on $\mathbf{W}$ with the above eigenvalues, and is therefore diagonalizable on $\mathbf{W}$. Our aim is to explicitly describe eigenvectors of $\mathcal{X}$ on $\mathbf{W}$. To this end, recall the Leonard systems $\Phi, \Phi^{\perp}$ on $M \hat{x}, M \hat{x}^{\perp}$ from Propositions 6.1 and 6.2, respectively, and observe that $\left\{E_{i} \hat{x}\right\}_{i=0}^{D},\left\{E_{i} u_{0}^{\perp}\right\}_{i=1}^{D-1}$ form $\Phi^{*}$ - and $\left(\Phi^{\perp}\right)^{*}$-standard bases for $M \hat{x}, M \hat{x}^{\perp}$, respectively; cf. (5.1). We will work with the following ordered orthogonal basis for $\mathbf{W}$ :

$$
\mathcal{B}=\left\{E_{0} \hat{x}, E_{1} \hat{x}, E_{1} u_{0}^{\perp}, E_{2} \hat{x}, E_{2} u_{0}^{\perp}, \ldots, E_{D-1} \hat{x}, E_{D-1} u_{0}^{\perp}, E_{D} \hat{x}\right\} .
$$

Recall the orthogonal projection $\pi$ (resp. $\widetilde{\pi}$ ) from $\mathbf{W}$ onto $M \hat{x}$ (resp. $M \hat{C})$.

Lemma 10.1. The following (i), (ii) hold:

(i) The matrix representing $\pi$ in $\mathcal{B}$ is

$$
\operatorname{blockdiag}(\pi(0), \pi(1), \ldots, \pi(D-1), \pi(D)),
$$

where $\pi(0)=\pi(D)=(1)$, and $\pi(i)=\operatorname{diag}(1,0)$ for $1 \leqslant i \leqslant D-1$.

(ii) The matrix representing $\widetilde{\pi}$ in $\mathcal{B}$ is

$$
\operatorname{blockdiag}(\tilde{\pi}(0), \widetilde{\pi}(1), \ldots, \widetilde{\pi}(D-1), \widetilde{\pi}(D)),
$$

where $\tilde{\pi}(0)=(1), \tilde{\pi}(D)=(0)$, and for $1 \leqslant i \leqslant D-1$,

$$
\begin{aligned}
\tilde{\pi}(i)= & \frac{1}{\left(q^{D}-1\right)\left(1+q^{e}\right)} \\
& \times\left(\begin{array}{cc}
\left(q^{e}+q^{i}\right)\left(q^{D-i}-1\right) & q^{-1}\left(1+q^{D+e-i}\right)\left(q^{i}-1\right)\left(q^{e}+q^{i}\right)\left(q^{D-i}-1\right) \\
q & \left(1+q^{D+e-i}\right)\left(q^{i}-1\right)
\end{array}\right) .
\end{aligned}
$$

Proof. (i) Immediate from $\pi E_{i} \hat{x}=E_{i} \hat{x}, 0 \leqslant i \leqslant D$, and $\pi E_{i} u_{0}^{\perp}=0,1 \leqslant i \leqslant D-1$.

(ii) We also recall the Leonard systems $\widetilde{\Phi}, \widetilde{\Phi}^{\perp}$ on $M \hat{C}, M \hat{C}^{\perp}$ from Propositions 7.1 and 7.2 , respectively, and consider the $\widetilde{\Phi}^{*}$ - and $\left(\widetilde{\Phi}^{\perp}\right)^{*}$-standard bases $\left\{E_{i} \hat{C}_{0}\right\}_{i=0}^{D-1}, \quad\left\{E_{i} \widetilde{u}_{0}^{\perp}\right\}_{i=1}^{D}$ for $M \hat{C}, M \hat{C}^{\perp}$, respectively. First, we obtain $\widetilde{\pi}(0)=(1)$ and $\widetilde{\pi}(D)=(0)$ since $E_{0} \hat{x} \in M \hat{C}$ and $E_{D} \hat{x} \in M \hat{C}^{\perp}$. Next, let $1 \leqslant i \leqslant D-1$. By (6.4), (7.1), and since $\hat{C}_{0}=\hat{C}_{0}^{-}+\hat{C}_{0}^{+}$, we have

$$
\hat{C}_{0}=\hat{x}+\frac{q-1}{q^{D}-1} A \hat{x}+\frac{q}{q^{D}-1} u_{0}^{\perp}, \quad \widetilde{u}_{0}^{\perp}=-q^{e} \hat{x}+\frac{q-1}{q^{D}-1} A \hat{x}+\frac{q}{q^{D}-1} u_{0}^{\perp} .
$$

Then, using (3.2) we have

$$
E_{i} \hat{C}_{0}=\left(1+\frac{q-1}{q^{D}-1} \theta_{i}\right) E_{i} \hat{x}+\frac{q}{q^{D}-1} E_{i} u_{0}^{\perp}=\frac{\left(q^{e}+q^{i}\right)\left(q^{D-i}-1\right)}{q^{D}-1} E_{i} \hat{x}+\frac{q}{q^{D}-1} E_{i} u_{0}^{\perp},
$$

and likewise we have

$$
E_{i} \widetilde{u}_{0}^{\perp}=\frac{\left(1+q^{D+e-i}\right)\left(1-q^{i}\right)}{q^{D}-1} E_{i} \hat{x}+\frac{q}{q^{D}-1} E_{i} u_{0}^{\perp} .
$$

Solving these equations for $E_{i} \hat{x}, E_{i} u_{0}^{\perp}$, it follows that

$$
\begin{aligned}
& E_{i} \hat{x}=\frac{1}{1+q^{e}} E_{i} \hat{C}_{0}-\frac{1}{1+q^{e}} E_{i} \widetilde{u}_{0}^{\perp}, \\
& E_{i} u_{0}^{\perp}=\frac{\left(1+q^{D+e-i}\right)\left(q^{i}-1\right)}{q\left(1+q^{e}\right)} E_{i} \hat{C}_{0}+\frac{\left(q^{e}+q^{i}\right)\left(q^{D-i}-1\right)}{q\left(1+q^{e}\right)} E_{i} \widetilde{u}_{0}^{\perp} .
\end{aligned}
$$

By these comments and since $\widetilde{\pi} E_{i} \hat{C}_{0}=E_{i} \hat{C}_{0}, \widetilde{\pi} E_{i} \widetilde{u}_{0}^{\perp}=0$, we obtain $\widetilde{\pi}(i)$ given above. 
Lemma 10.2. The matrix representing $\mathcal{X}$ on $\mathbf{W}$ in $\mathcal{B}$ is

$\operatorname{blockdiag}(x(0), x(1), \ldots, x(D-1), x(D))$,

where $x(0)=(\tau), x(D)=\left(\tau^{-1} q^{-D}\right)$, and for $1 \leqslant i \leqslant D-1$,

$$
x(i)=\frac{\tau}{q^{D}-1}\left(\begin{array}{cc}
q^{D+e-i}-q^{D+e}+q^{D}-q^{i} & q^{-1}\left(1+q^{D+e-i}\right)\left(q^{i}-1\right)\left(q^{e}+q^{i}\right)\left(q^{D-i}-1\right) \\
q^{D+1} & -q^{2 D+e-i}+q^{D+i}+q^{D+e}-q^{D}
\end{array}\right) .
$$

Proof. Since $X=\mathcal{T}^{\prime} \mathcal{T}$, the result routinely follows from Theorem 8.14 and Lemma 10.1.

Define the vectors $\mathbf{y}_{i},-D \leqslant i \leqslant D-1$, by

$$
\begin{aligned}
& \mathbf{y}_{i}=\frac{\left(q^{D-i}-1\right)\left(1+q^{D+e-i}\right)}{\left(q^{D}-1\right)\left(1+q^{D+e-2 i}\right)} E_{i} \hat{x}+\frac{q^{D-i+1}}{\left(q^{D}-1\right)\left(1+q^{D+e-2 i}\right)} E_{i} u_{0}^{\perp}, \\
& \mathbf{y}_{-i}=\frac{q^{D-2 i}\left(q^{i}-1\right)\left(q^{e}+q^{i}\right)}{\left(q^{D}-1\right)\left(1+q^{D+e-2 i}\right)} E_{i} \hat{x}-\frac{q^{D-i+1}}{\left(q^{D}-1\right)\left(1+q^{D+e-2 i}\right)} E_{i} u_{0}^{\perp}
\end{aligned}
$$

for $1 \leqslant i \leqslant D-1$, and

$$
\mathbf{y}_{0}=E_{0} \hat{x}, \quad \mathbf{y}_{-D}=E_{D} \hat{x} .
$$

Observe that the $\mathbf{y}_{i}$ are real vectors. We normalized the $\mathbf{y}_{i}$ so that

$$
\mathbf{y}_{i}+\mathbf{y}_{-i}=E_{i} \hat{x}, \quad 1 \leqslant i \leqslant D-1 .
$$

Proposition 10.3. For $-D \leqslant i \leqslant D-1, \mathbf{y}_{i}$ is an eigenvector of $\mathcal{X}$ on $\mathbf{W}$ with eigenvalue $\lambda_{i}$. Moreover, we have $\sum_{i=-D}^{D-1} \mathbf{y}_{i}=\hat{x}$.

Proof. The first assertion follows from direct computations using Lemma 10.2. By (10.2), we have

$$
\sum_{i=-D}^{D-1} \mathbf{y}_{i}=\mathbf{y}_{0}+\sum_{i=1}^{D-1}\left(\mathbf{y}_{i}+\mathbf{y}_{-i}\right)+\mathbf{y}_{-D}=\sum_{i=0}^{D} E_{i} \hat{x}=\hat{x}
$$

as desired.

By Proposition 10.3, the $\mathbf{y}_{i}$ form an eigenbasis of $\mathcal{X}$ on $\mathbf{W}$. Since $\mathcal{B}$ is an orthogonal basis for $\mathbf{W}$, it follows that $\left\langle\mathbf{y}_{i}, \mathbf{y}_{j}\right\rangle \neq 0$ implies $j \in\{i,-i\}$. We now compute these inner products.

Lemma 10.4. The following $(i)$, (ii) hold:

(i) For $0 \leqslant i \leqslant D$, we have

$$
\left\|E_{i} \hat{x}\right\|^{2}=(-1)^{i} q^{i(D+e-i+1)} \frac{\left(q^{-D} ; q\right)_{i}\left(1+q^{D+e-2 i}\right)}{(q ; q)_{i}\left(-q^{e-i} ; q\right)_{D+1}} .
$$

(ii) For $1 \leqslant i \leqslant D-1$, we have

$$
\left\|E_{i} u_{0}^{\perp}\right\|^{2}=(-1)^{i-1} q^{i(D+e-i+1)+D-2} \frac{\left(q^{-D} ; q\right)_{i+1}\left(1+q^{D+e-2 i}\right)}{(q ; q)_{i-1}\left(-q^{e-i+1} ; q\right)_{D-1}} .
$$


Proof. Let $m_{i}$ (resp. $m_{i}^{\perp}$ ) denote the scalar $\mathrm{m}_{i}$ from (5.8) for the Leonard system $\Phi$ (resp. $\Phi^{\perp}$ ).

(i) Since $\left\|E_{i} \hat{x}\right\|^{2}=\left\langle\hat{x}, E_{0}^{*} E_{i} E_{0}^{*} \hat{x}\right\rangle=m_{i}$, the result follows from Proposition 6.1 and (5.11).

(ii) By (6.2) and Lemma 4.4, we have

$$
\left\|u_{0}^{\perp}\right\|^{2}=q^{e-1}\left(q^{D-1}-1\right)\left(q^{D}-1\right) .
$$

Since $\left\|E_{i} u_{0}^{\perp}\right\|^{2}=m_{i-1}^{\perp}\left\|u_{0}^{\perp}\right\|^{2}$ in this case, the result follows from Proposition 6.2 and (5.11).

Lemma 10.5. For $1 \leqslant i \leqslant D-1$, we have

$$
\begin{aligned}
& \left\|\mathbf{y}_{i}\right\|^{2}=(-1)^{i} q^{i(D+e-i)} \frac{\left(q^{1-D} ; q\right)_{i}\left(1+q^{D}+q^{D+e-i}-q^{D-i}\right)}{(q ; q)_{i}\left(-q^{e-i} ; q\right)_{D}\left(1+q^{D+e-2 i}\right)} \\
& \left\|\mathbf{y}_{-i}\right\|^{2}=(-1)^{i-1} q^{i(D+e-i-2)+D+e} \frac{\left(q^{1-D} ; q\right)_{i-1}\left(1+q^{D}+q^{i-e}-q^{i}\right)}{(q ; q)_{i-1}\left(-q^{e-i+1} ; q\right)_{D}\left(1+q^{D+e-2 i}\right)} \\
& \left\langle\mathbf{y}_{i}, \mathbf{y}_{-i}\right\rangle=(-1)^{i} q^{i(D+e-i-1)+D} \frac{\left(q^{1-D} ; q\right)_{i}}{(q ; q)_{i-1}\left(-q^{e-i+1} ; q\right)_{D-1}\left(1+q^{D+e-2 i}\right)}
\end{aligned}
$$

Moreover,

$$
\left\|\mathbf{y}_{0}\right\|^{2}=\frac{1}{\left(-q^{e} ; q\right)_{D}}, \quad\left\|\mathbf{y}_{-D}\right\|^{2}=\frac{1}{\left(-q^{-e} ; q\right)_{D}} .
$$

Proof. The above values are routinely computed using Lemma 10.4.

Proposition 10.6. For $f, g \in \mathfrak{L}$, we have

$$
\langle f(X) . \hat{x}, g(X) . \hat{x}\rangle=\sum_{i=-D}^{D-1} f\left(\lambda_{i}\right) \overline{g\left(\lambda_{i}\right)}\left\|\mathbf{y}_{i}\right\|^{2}+\sum_{i=1}^{D-1}\left(f\left(\lambda_{i}\right) \overline{g\left(\lambda_{-i}\right)}+f\left(\lambda_{-i}\right) \overline{g\left(\lambda_{i}\right)}\right)\left\langle\mathbf{y}_{i}, \mathbf{y}_{-i}\right\rangle .
$$

Proof. From Lemma 10.3 it follows that

$$
f(X) . \hat{x}=f(X) \cdot \sum_{i=-D}^{D-1} \mathbf{y}_{i}=\sum_{i=-D}^{D-1} f\left(\lambda_{i}\right) \mathbf{y}_{i},
$$

and similarly for $g(X) . \hat{x}$. Since the $\mathbf{y}_{i}$ are real, the result is immediate from this comment.

Define the Hermitian form $\langle\cdot, \cdot\rangle_{\mathfrak{L}}$ on the subspace $\mathfrak{L}$ from $(9.1)$ by $^{13}$

$$
\langle f, g\rangle_{\mathfrak{L}}=\sum_{i=-D}^{D-1} f\left(\lambda_{i}\right) \overline{g\left(\lambda_{i}\right)}\left\|\mathbf{y}_{i}\right\|^{2}+\sum_{i=1}^{D-1}\left(f\left(\lambda_{i}\right) \overline{g\left(\lambda_{-i}\right)}+f\left(\lambda_{-i}\right) \overline{g\left(\lambda_{i}\right)}\right)\left\langle\mathbf{y}_{i}, \mathbf{y}_{-i}\right\rangle
$$

for $f, g \in \mathfrak{L}$, where the $\lambda_{i}$ are from (10.1), and the $\left\|\mathbf{y}_{i}\right\|^{2}$ and the $\left\langle\mathbf{y}_{i}, \mathbf{y}_{-i}\right\rangle$ are given in Lemma 10.5. Since $\langle f, f\rangle_{\mathfrak{L}}=0$ implies $f \in\left(p^{\perp} h_{D-1}^{\perp} \mathbb{C}\left[\eta, \eta^{-1}\right]\right) \cap \mathfrak{L}=0$, it follows that $\langle\cdot, \cdot\rangle_{\mathfrak{L}}$ is an inner product on $\mathfrak{L}$. From (10.2) it also follows that, when restricted to symmetric polynomials, (10.3) gives an inner product for which the (symmetric) dual $q$-Krawtchouk polynomials (5.10) for the Leonard system $\Phi$ are orthogonal; cf. [14, Section 14.17], [35, Section 16].

We now present the orthogonality relation for the non-symmetric dual $q$-Krawtchouk polynomials $\ell_{i}^{ \pm}$from Definition 9.2:

Theorem 10.7. With reference to the inner product (10.3), we have

$$
\left\langle\ell_{i}^{\mu}, \ell_{j}^{\nu}\right\rangle_{\mathfrak{L}}=\delta_{i, j} \delta_{\mu, \nu}\left|C_{i}^{\mu}\right|
$$

for $0 \leqslant i, j \leqslant D-1$ and $\mu, \nu \in\{+,-\}$, where the $\left|C_{i}^{ \pm}\right|$are given in Lemma 4.4 .

Proof. Immediate from Propositions 9.1 and 10.6.

\footnotetext{
${ }^{13}$ We would like to point out here that the terms involving the $\left\langle\mathbf{y}_{i}, \mathbf{y}_{-i}\right\rangle$ are missing in the definition of the corresponding Hermitian form for the non-symmetric $q$-Racah polynomials given in [17, equation (77)]. A corrected statement will be given elsewhere.
} 


\section{Acknowledgements}

The authors thank Daniel Orr for helpful comments on nil-DAHAs of type $\left(C_{n}^{\vee}, C_{n}\right)$. They also thank Paul Terwilliger for many valuable discussions, Marta Mazzocco and Alexei Zhedanov for bringing the authors' attention to [21, 22], and the anonymous referees for carefully reading the paper. Part of this work was done while Jae-Ho Lee was visiting Tohoku University as a JSPS Postdoctoral Fellow. Hajime Tanaka was supported by JSPS KAKENHI Grant Numbers JP25400034 and JP17K05156. An extended abstract of this work appeared in the proceedings of FPSAC'17, London, UK, July 2017; cf. [18].

\section{References}

[1] Bannai E., Ito T., Algebraic combinatorics. I. Association schemes, The Benjamin/Cummings Publishing Co., Inc., Menlo Park, CA, 1984.

[2] Brouwer A.E., Cohen A.M., Neumaier A., Distance-regular graphs, Ergebnisse der Mathematik und ihrer Grenzgebiete (3), Vol. 18, Springer-Verlag, Berlin, 1989.

[3] Cherednik I., Double affine Hecke algebras, Knizhnik-Zamolodchikov equations, and Macdonald's operators, Int. Math. Res. Not. 1992 (1992), 171-180.

[4] Cherednik I., Double affine Hecke algebras and Macdonald's conjectures, Ann. of Math. 141 (1995), 191216.

[5] Cherednik I., Macdonald's evaluation conjectures and difference Fourier transform, Invent. Math. 122 (1995), 119-145, q-alg/9503006.

[6] Cherednik I., Nonsymmetric Macdonald polynomials, Int. Math. Res. Not. 1995 (1995), 483-515, q-alg/9505029.

[7] Cherednik I., Orr D., One-dimensional nil-DAHA and Whittaker functions I, Transform. Groups 17 (2012), 953-987, arXiv:1104.3918.

[8] Cherednik I., Orr D., One-dimensional nil-DAHA and Whittaker functions II, Transform. Groups 18 (2013), 23-59, arXiv:1104.3918.

[9] Cherednik I., Orr D., Nonsymmetric difference Whittaker functions, Math. Z. 279 (2015), 879-938, arXiv:1302.4094.

[10] Van Dam E.R., Koolen J.H., Tanaka H., Distance-regular graphs, Electron. J. Combin. (2016), \#DS22, 156 pages, arXiv:1410.6294.

[11] Godsil C.D., Algebraic combinatorics, Chapman and Hall Mathematics Series, Chapman \& Hall, New York, 1993.

[12] Hosoya R., Suzuki H., Tight distance-regular graphs with respect to subsets, European J. Combin. 28 (2007), $61-74$.

[13] Ito T., Tanabe K., Terwilliger P., Some algebra related to $P$ - and $Q$-polynomial association schemes, in Codes and Association Schemes (Piscataway, NJ, 1999), DIMACS Ser. Discrete Math. Theoret. Comput. Sci., Vol. 56, Amer. Math. Soc., Providence, RI, 2001, 167-192, math.CO/0406556.

[14] Koekoek R., Lesky P.A., Swarttouw R.F., Hypergeometric orthogonal polynomials and their $q$-analogues, Springer Monographs in Mathematics, Springer-Verlag, Berlin, 2010.

[15] Koornwinder T.H., Bouzeffour F., Nonsymmetric Askey-Wilson polynomials as vector-valued polynomials, Appl. Anal. 90 (2011), 731-746, arXiv:1006.1140.

[16] Lee J.-H., Q-polynomial distance-regular graphs and a double affine Hecke algebra of rank one, Linear Algebra Appl. 439 (2013), 3184-3240, arXiv:1307.5297.

[17] Lee J.-H., Nonsymmetric Askey-Wilson polynomials and Q-polynomial distance-regular graphs, J. Combin. Theory Ser. A 147 (2017), 75-118, arXiv:1509.04433.

[18] Lee J.-H., Tanaka H., Dual polar graphs, a nil-DAHA of rank one, and non-symmetric dual q-Krawtchouk polynomials, Sém. Lothar. Combin. 78B (2017), Art. 42, 12 pages.

[19] Leonard D.A., Orthogonal polynomials, duality and association schemes, SIAM J. Math. Anal. 13 (1982), 656-663. 
[20] Macdonald I.G., Affine Hecke algebras and orthogonal polynomials, Cambridge Tracts in Mathematics, Vol. 157, Cambridge University Press, Cambridge, 2003.

[21] Mazzocco M., Non-symmetric basic hypergeometric polynomials and representation theory for confluent Cherednik algebras, SIGMA 10 (2014), 116, 10 pages, arXiv:1409.4287.

[22] Mazzocco M., Confluences of the Painlevé equations, Cherednik algebras and q-Askey scheme, Nonlinearity 29 (2016), 2565-2608, arXiv:1307.6140.

[23] Nomura K., Terwilliger P., The universal DAHA of type $\left(C_{1}^{\vee}, C_{1}\right)$ and Leonard pairs of $q$-Racah type, Linear Algebra Appl. 533 (2017), 14-83, arXiv:1701.06089.

[24] Oblomkov A., Stoica E., Finite dimensional representations of the double affine Hecke algebra of rank 1, J. Pure Appl. Algebra 213 (2009), 766-771, math.RT/0409256.

[25] Sahi S., Nonsymmetric Koornwinder polynomials and duality, Ann. of Math. 150 (1999), 267-282, q-alg/9710032.

[26] Stanton D., Some q-Krawtchouk polynomials on Chevalley groups, Amer. J. Math. 102 (1980), 625-662.

[27] Stanton D., Three addition theorems for some $q$-Krawtchouk polynomials, Geom. Dedicata 10 (1981), 403425.

[28] Suzuki H., The Terwilliger algebra associated with a set of vertices in a distance-regular graph, J. Algebraic Combin. 22 (2005), 5-38.

[29] Tanaka H., A bilinear form relating two Leonard systems, Linear Algebra Appl. 431 (2009), 1726-1739, arXiv:0807.0385.

[30] Tanaka H., Vertex subsets with minimal width and dual width in $Q$-polynomial distance-regular graphs, Electron. J. Combin. 18 (2011), no. 1, \#P167, 32 pages, arXiv:1011.2000.

[31] Terwilliger P., The subconstituent algebra of an association scheme. I, J. Algebraic Combin. 1 (1992), 363388.

[32] Terwilliger P., The subconstituent algebra of an association scheme. II, J. Algebraic Combin. 2 (1993), 73-103.

[33] Terwilliger P., The subconstituent algebra of an association scheme. III, J. Algebraic Combin. 2 (1993), $177-210$.

[34] Terwilliger P., Two linear transformations each tridiagonal with respect to an eigenbasis of the other, Linear Algebra Appl. 330 (2001), 149-203, math.RA/0406555.

[35] Terwilliger P., Leonard pairs and the q-Racah polynomials, Linear Algebra Appl. 387 (2004), 235-276, math.QA/0306301.

[36] Terwilliger P., Two linear transformations each tridiagonal with respect to an eigenbasis of the other; comments on the parameter array, Des. Codes Cryptogr. 34 (2005), 307-332, math.RA/0306291.

[37] Worawannotai C., Dual polar graphs, the quantum algebra $U_{q}\left(\mathfrak{s l}_{2}\right)$, and Leonard systems of dual $q$ Krawtchouk type, Linear Algebra Appl. 438 (2013), 443-497, arXiv:1205.2144. 